CLARISSA WILLETS BEZERRA

\title{
Associação entre percepção sobre características de vizinhança e aterosclerose subclínica: um estudo transversal no ELSA-Brasil
}

Tese apresentada à Faculdade de Medicina da Universidade de São Paulo para obtenção do título de Doutora em Ciências

Programa de Fisiopatologia Experimental

Orientadora: Profa. Dra. Claudia Kimie Suemoto

São Paulo 
CLARISSA WILLETS BEZERRA

CLARISSA WILLETS BEZERRA

\title{
Associação entre percepção sobre características de vizinhança e aterosclerose subclínica: um estudo transversal no ELSA-Brasil
}

\author{
Tese apresentada à Faculdade de Medicina da \\ Universidade de São Paulo para obtenção do título \\ de Doutora em Ciências \\ Programa de Fisiopatologia Experimental \\ Orientadora: Profa. Dra. Claudia Kimie Suemoto
}

(Versão corrigida. Resolução CoPGr 6018, de 06 de outubro de 2011.

A versão original está disponível na Biblioteca FMUSP)

São Paulo

2019 
Dados Internacionais de Catalogação na Publicação (CIP)

Preparada pela Biblioteca da

Faculdade de Medicina da Universidade de São Paulo

Creprodução autorizada pelo autor

Bezerra, Clarissa Willets

Associação entre percepção sobre características de vizinhança e aterosclerose subclínica : um estudo transversal no ELSA-Brasil / Clarissa Willets Bezerra. -- São Paulo, 2019.

Tese(doutorado)--Faculdade de Medicina da Universidade de São Paulo.

Programa de Fisiopatologia Experimental.

Orientadora: Claudia Kimie Suemoto.

Descritores: 1.Aterosclerose 2.Características de residência 3. Artérias carótidas 4.Espessura íntimamédia carotídea 5.Atividade motora 6.Exposição à violência

$\mathrm{USP} / \mathrm{FM} / \mathrm{DBD}-510 / 19$

Responsável: Erinalva da Conceição Batista, CRB-8 6755 
Dedico este trabalho a meus filhos, Caio e Gabriel, gestados junto com esta tese. Que eu sempre possa inspirá-los a serem fortes e generosos com o mundo.

E à minha mãe, Lillian, por sempre estar ao meu lado e cuidar de tudo para que eu possa realizar meus sonhos e ser livre. 


\section{Agradecimentos}

À Profa. Claudia Suemoto, que me alfabetizou com paciência no campo da pesquisa e que, principalmente, me acolheu com generosidade em todos os momentos da elaboração deste trabalho.

À Profa. Isabela Benseñor, pelas contribuições valiosas ao longo dessa jornada e por ter me aberto as portas do ELSA-Brasil, apostando neste projeto.

Ao Prof. Paulo Lotufo, também acreditou em nós e nos incentivou desde a primeira reunião do ELSA-Brasil. Esse trabalho não seria possível sem sua confiança.

Ao Prof. Itamar Santos, que tanto contribui para minha formação como médica e pesquisadora, da ausculta cardíaca à interpretação do p-valor. Obrigada pelas contribuições que iluminaram este estudo.

À Vivian Avelino da Silva, pela sua presença marcante em toda minha formação, com orientações e direcionamentos, por ter confiado em mim e ter me conectando à Profa. Claudia.

Ao Médico de Família e Comunidade Carlos Frederico Confort Campos, amigo querido e incentivador incansável de meu crescimento pessoal e profissional, entre cafés e sobremesas. 
Ao médico de Família e Comunidade Marco Antônio Santos, que me ensinou que geografia e Medicina de Família e Comunidade estão muito mais próximos do que eu imaginava e por servir de prumo todos os dias.

Às Enfermeiras e amigas Ana Claudia Pastori e lara Oliveira Lopes por terem me ensinado o a cuidar de pessoas vulneráveis naquela salinha apertada e cheia de amor na UBS Jd. Boa Vista.

Ao meu tio, Henrique Cabrita, primeiro a despertar em mim a curiosidade e o amor pela medicina e por ter me incentivado a sempre buscar o melhor.

Ao meu companheiro, João Renato Negrão, quem eu escolhi para realizar meus sonhos.

À Ingrit Bianca Britto, que cuida dos meus filhos com todo seu amor, atenção e imensa disposição, o que é um privilégio e por quem serei eternamente grata.

Ao Tchelo Pires, avô dos meus filhos, pelas dicas na redação deste produto e por ter aceitado o cargo de avô com doçura, inclusive nas manhãs de sábado, para que eu pudesse escrever.

A todos os participantes do ELSA-Brasil, que disponibilizam seu tempo e presença para contribuir com a ciência. 
"Tudo, tudo, tudo, tudo que nóis tem é nóis"

Emicida 
Esta tese está de acordo com as seguintes normas, em vigor no momento desta publicação:

Referências: adaptado de International Committee of Medical Journals Editors (Vancouver).

Universidade de São Paulo. Faculdade de Medicina. Divisão de Biblioteca e Documentação. Guia de apresentação de dissertações, teses e monografias. Elaborado por Anneliese Carneiro da Cunha, Maria Julia de A. L. Freddi, Maria F. Crestana, Marinalva de Souza Aragão, Suely Campos Cardoso, Valéria Vilhena. $3 a$ ed. São Paulo: Divisão de Biblioteca e Documentação; 2011.

Abreviaturas dos títulos dos periódicos de acordo com List of Journals Indexed in Index Medicus. 


\section{Sumário}

Lista de Tabelas

Lista de Figuras

Lista de Siglas

Resumo

Summary

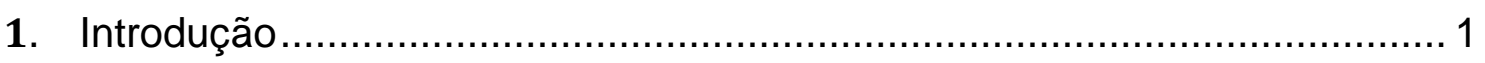

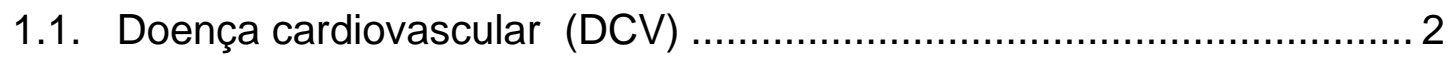

1.1.1. Doença cardiovascular no Brasil ................................................ 3

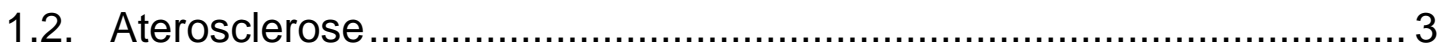

1.3. Aterosclerose subclínica: a espessura íntima-média de artérias

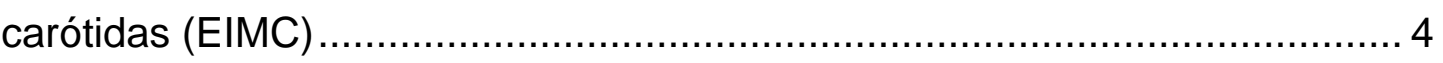

1.4. Estratégias de prevenção populacionais e estudos de vizinhança ......... 6

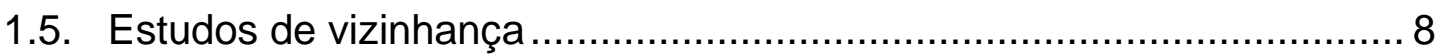

1.5.1. Estudos de vizinhança, DCV e aterosclerose subclínica ................ 8

Características dos estudos de vizinhança e DCV.............................. 10

Diferenças entre os sexos..................................................... 13

Medidas de vizinhança por questionários ........................................ 14

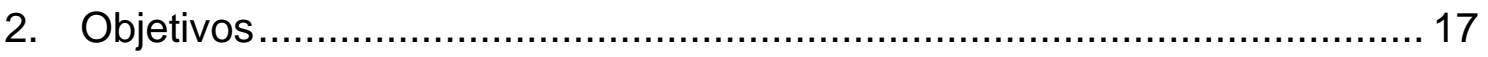

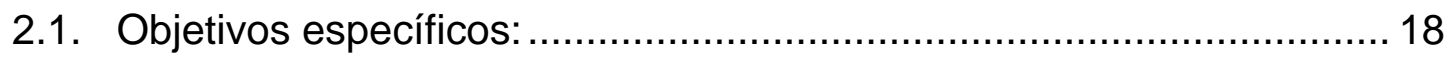

2.2. Hipótese ................................................................................... 18

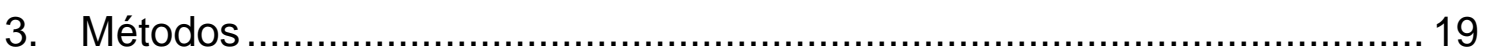




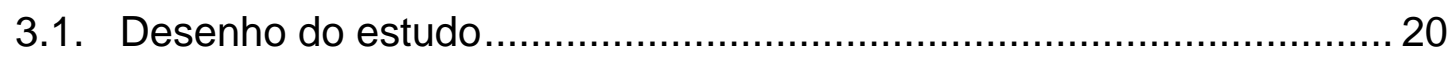

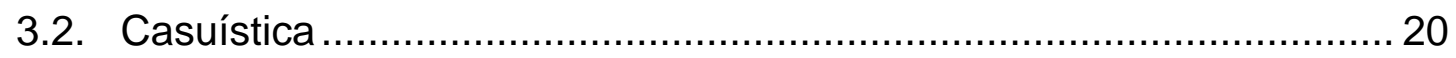

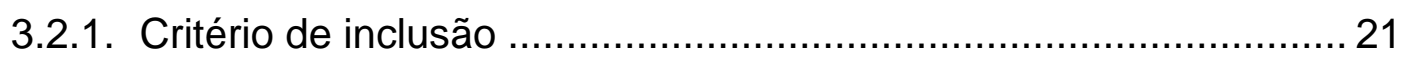

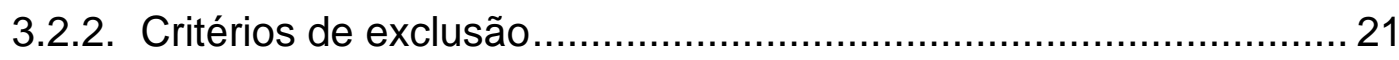

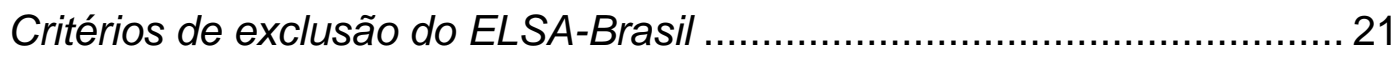

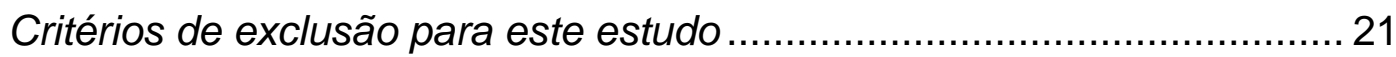

3.3. Variável preditora: questionário da percepção sobre características de

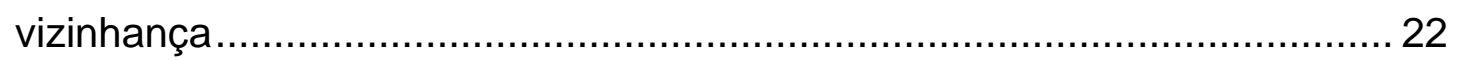

3.4. Variável de desfecho: espessura íntima-média de carótidas............... 24

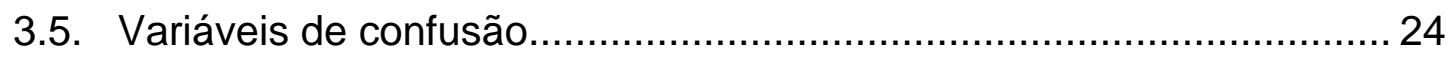

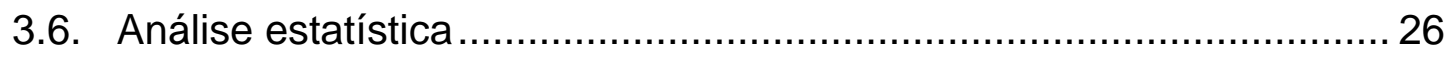

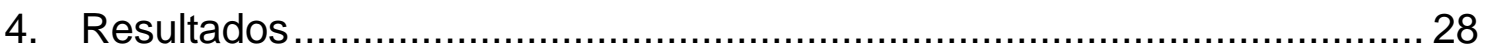

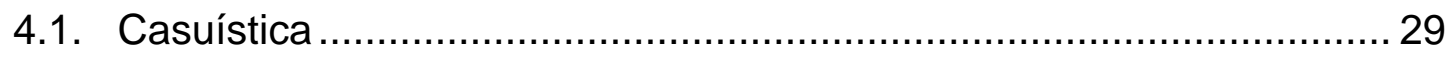

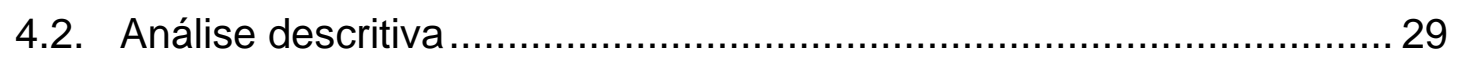

4.3. Associação entre EIMC e percepção de vizinhança .............................. 34

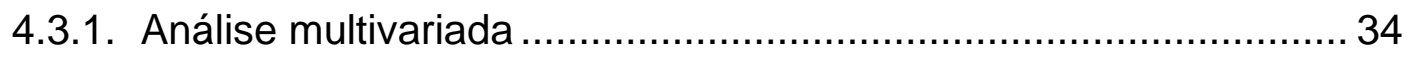

4.3.2. Análise estratificada por anos de residência ................................. 37

4.3.3. Análise de interação entre percepção de vizinhança e sexo ......... 39

4.3.4. Análise por método de imputação múltipla .................................... 40

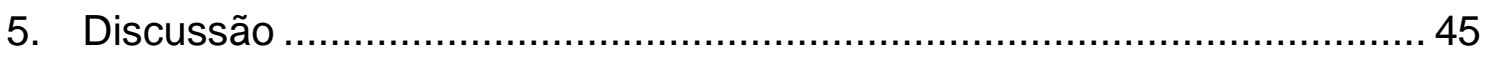

5.1. Comparação com resultados de estudos anteriores ............................ 46

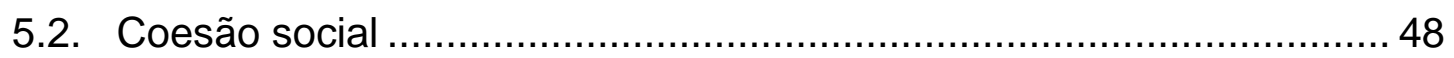


5.3. Ambiente para atividade física .................................................. 49

5.4. Disponibilidade de alimento saudável....................................... 50

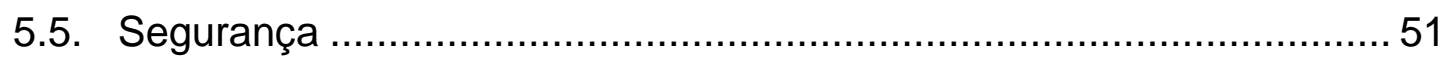

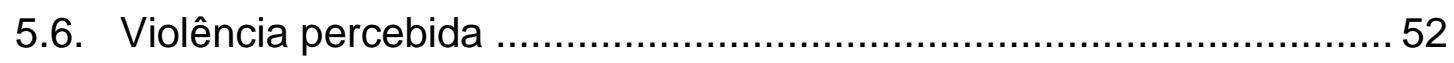

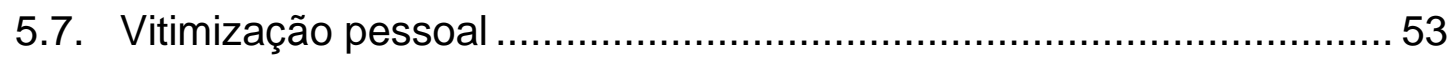

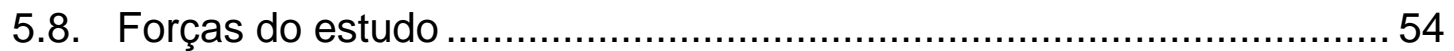

5.9. Limitações do estudo ......................................................... 55

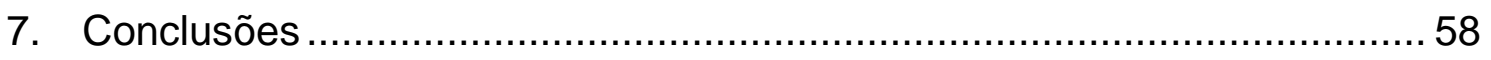

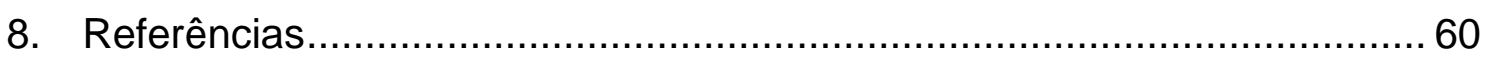




\section{Lista de Tabelas}

Tabela 1 - Associação entre EIMC $(\mu \mathrm{m})$ e variáveis demográficas e clínicas, ajustado por idade e sexo $(n=9.923)$

Tabela 2 - Percepção de características de vizinhança $(n=9.923)$ 33

Tabela 3 - Coeficientes e intervalos de confiança (IC) de 95\% para EIMC $(\mu \mathrm{m})$, de acordo com percepção de características de vizinhança $(n=9.923)$..... 36

Tabela 4 - Coeficientes e intervalos de confiança (IC) de 95\% para EIMC $(\mu \mathrm{m})$ de acordo com percepção de características de vizinhança, estratificado por anos de residência $(n=9.923)$.

Tabela 5 - Coeficientes e intervalos de confiança (IC) de 95\% para EIMC $(\mu \mathrm{m})$ de acordo com percepção de características de vizinhança, estratificado por $\operatorname{sexo}(n=9.923)$

Tabela 6 - Coeficientes e intervalos de confiança (IC) de 95\% para EIMC ( $\mu \mathrm{m})$ de acordo com percepção de características de vizinhança $(n=9.923)$ 42

Tabela 7 - Coeficientes e intervalos de confiança (IC) de 95\% para EIMC $(\mu \mathrm{m})$ de acordo com percepção de características de vizinhança, estratificado por anos de residência $(n=9.923)$ 


\section{Lista de Figuras}

Figura 1 - Representação gráfica das causas de morte globais no ano de 2016

Figura 2 - Estratégias de prevenção populacional e de alto risco, segundo

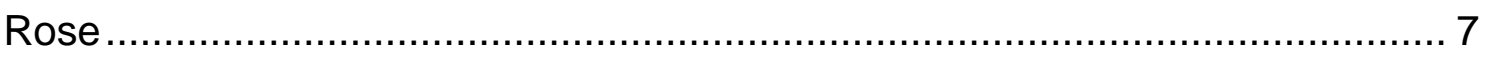

Figura 3 - Esquema conceitual das relações hipotéticas entre ambientes de vizinhança e DCV.

Figura 4 - Fluxograma dos participantes 29

Figura 5 - Histograma da pontuação dos participantes em cada domínio do questionário

Figura 6 - Fluxograma de participantes para o modelo de imputação múltipla para valores ausentes ("missings") da espessura íntima-média de carótidas .. 40 


\section{Lista de Siglas}

ARIC Atherosclerosis Risk in Communities

AVC Acidente vascular cerebral

CARDIA Coronary Artery Disease Risk Development in Young Adults

DATASUS Departamento de informática do Sistema Único de Saúde

DCNT Doenças crônicas não transmissíveis

DCV Doença cardiovascular

EIMC Espessura íntima-média de carótidas

ELSA-Brasil $\quad$ Estudo Longitudinal de Saúde do Adulto - Brasil

IC $95 \% \quad$ Intervalo de confiança de $95 \%$

MESA Multi-Ethnic Study of Atherosclerosis 


\section{Resumo}

Bezerra CW. Associação entre características de vizinhança e aterosclerose subclínica: um estudo transversal no ELSA-Brasil [tese]. São Paulo: Faculdade de Medicina, Universidade de São Paulo; 2019.

Introdução: Viver em uma vizinhança com contexto socioeconômico desfavorável pode aumentar o risco de doença coronariana. Entretanto, o impacto das características da vizinhança na aterosclerose subclínica e seu papel nos fatores de risco cardiovasculares ainda não foi estudado em países pobres. Objetivo Este estudo pesquisou a associação entre percepção de características de vizinhança e a prevalência de aterosclerose subclínica em uma grande amostra de adultos, utilizando dados transversais do ELSA-Brasil. Métodos: Participantes livres de doença cardiovascular clínica responderam a um questionário desenhado para acessar suas percepções acerca de seis domínios sobre características de sua vizinhança: coesão social, disponibilidade de alimento saudável, ambiente para atividade física, segurança, violência percebida e vitimização pessoal. A pontuação de cada domínio foi utilizada como variável independente. A aterosclerose subclínica foi medida pela EIMC (variável dependente). Modelos de regressão linear ajustados para variáveis demográficas, socioeconômicas e cardiovasculares foram utilizados para verificar a associação. Resultados: Foram examinados 9.923 participantes (média de idade $=51,5 \pm 8,9$ anos, $44 \%$ homens, $55 \%$ brancos). $\mathrm{Na}$ análise ajustada para idade, sexo e anos de residência, melhor percepção de ambiente para atividade física $(\beta=-0,62$, intervalo de confiança de $95 \%$ [IC 95\%] $=-1,01 ;-0,23, p=0,002)$ e menor violência percebida $(\beta=-$ 1,95, IC 95\% = -2,96; -0,94, $p<0,001)$ mostraram associação com menor EIMC. Curiosamente, baixa percepção de vitimização pessoal esteve associada com maiores EIMC ( $\beta=10,70$, IC 95\%=4,55; 16.85, $p=0,001)$. Uma interação limítrofe entre sexo e dois domínios foi encontrada: melhor coesão social foi associada a maior EIMC entre mulheres e melhor segurança foi associada a menor EIMC entre homens. Análise estratificada por tempo de residência e modelos de imputação múltipla reforçaram os resultados. Conclusão: A percepção de morar em local com melhor ambiente para atividade física e menor violência percebida na vizinhança foram associados à menor EIMC. Menor nível de estresse psicossocial e menor sedentarismo podem explicar a proteção.

Descritores: Aterosclerose; Características de residência; Artérias carótidas; Espessura íntima-média carotídea; Atividade motora; Exposição à violência. 


\section{Summary}

Bezerra CW. Association between perceived neighborhood characteristics and carotid intima-media thickness: cross sectional results from the ELSA-Brasil study [thesis]. São Paulo: "Faculdade de Medicina, Universidade de São Paulo"; 2019.

Background: Living in a neighborhood with a low socioeconomic context may increase the risk of coronary heart disease. However, few studies have explored the impact of neighborhood characteristics on subclinical atherosclerosis, and their role as a risk factor for cardiovascular disease has not yet been studied in poor countries. Objectives: This study aimed to investigate the association between perceived neighborhood characteristics and subclinical atherosclerosis in a large sample of adults, using cross-sectional data from the Brazilian Longitudinal Study of Adult Health (ELSA-Brasil). Methods: Participants free of clinical cardiovascular disease answered a questionnaire designed to assess their perceptions regarding six of their neighborhood dimensions: social cohesion, walkability, availability of healthy food, safety, witnessed violence, and personal victimization. The scores of each domain were used as independent variables. Common carotid intima-media thickness (CCIMT) was used as a dependent variable. Then linear regression models, adjusted by demographic, socioeconomic, and cardiovascular factors were used. Results: A total of 9,923 adults (mean age $=51.5 \pm 8.9$ years, $44 \%$ male, $55 \%$ white) were examined. In the univariate analysis, better walkability $(\beta=-$ $0.62,95 \%$ confidence interval [Cl]: -1.01 to $-0.23 ; p=0.002$ ) and low witnessed violence ( $\beta=-1.95 ; 95 \% \mathrm{Cl}:-2.96$ to $-0.94 ; p<0.0001)$ were associated with lower CCIMT, whereas low personal victimization was associated with higher CCIMT ( $\beta=10.70 ; 95 \% \mathrm{Cl}$ : 4.55 to $16.85 ; p=0.001$ ). A borderline interaction between neighborhood domain and sex was found. Better social cohesion may be associated with larger CCIMT among women, whereas better safety was associated with lower CCIMT among men. Multiple imputation for missing CCIMT data showed similar results. Conclusions: The perception of living in a more walkable environment and in a neighborhood where people witness fewer violent episodes can provide protection on the early phases of the atherosclerosis process. Less stress and the practice of physical activity may explain the protection.

Descriptors: Atherosclerosis; Residence characteristics; Carotid arteries; Carotid artery intima-media thickness; Motor activity; Exposure to violence. 
1. Introdução 


\subsection{Doença cardiovascular (DCV)}

As doenças cardiovasculares são a principal causa de morte no mundo. Mais pessoas morrem por essas enfermidades do que por qualquer outra causa. Segundo a Organização Pan-Americana de Saúde, no ano de 2016, estima-se que mais de 17 milhões de pessoas morreram por doenças cardiovasculares, o que representa $44 \%$ das mortes por doenças crônicas não transmissíveis (DCNT) e 31\% de todas as mortes em nível global (Figura 1) (1).

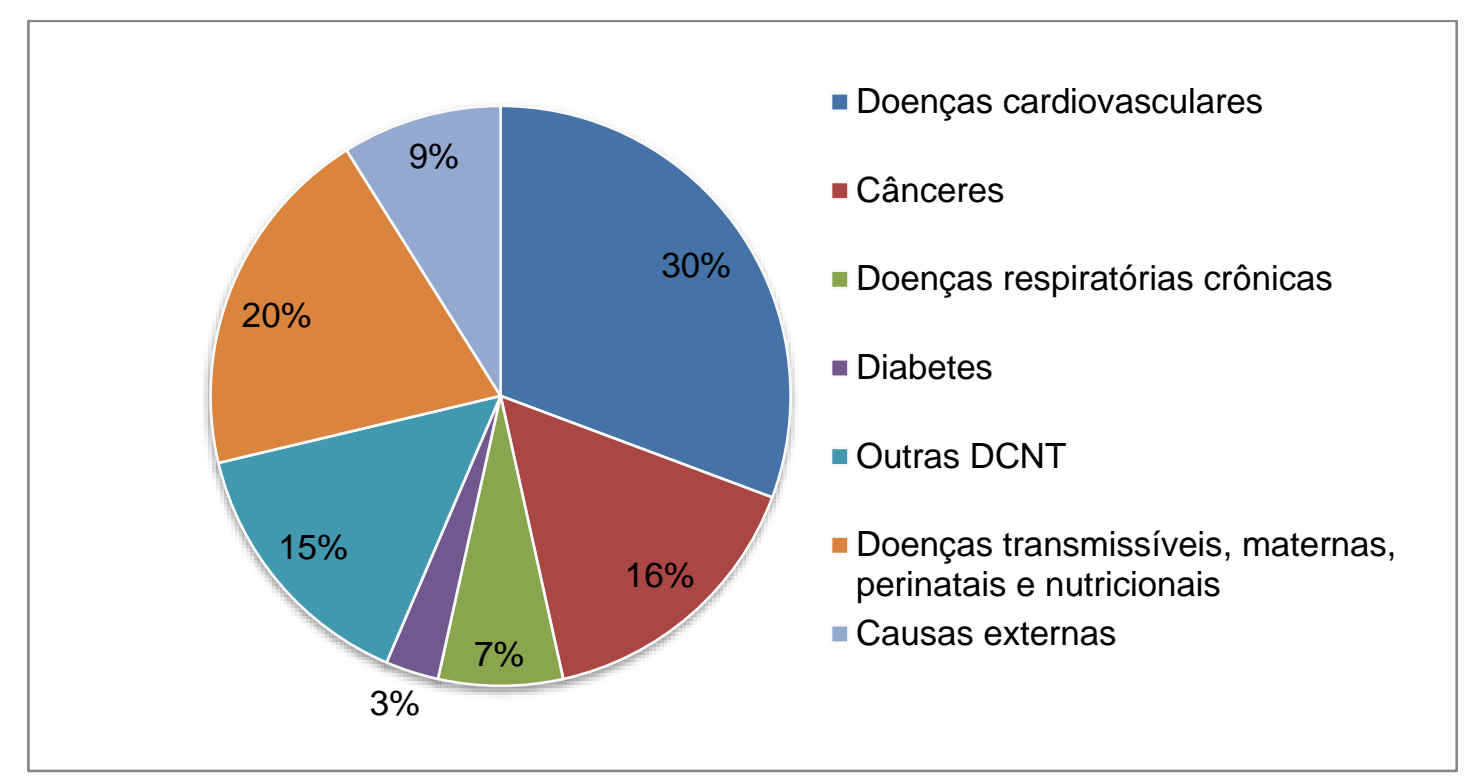

Figura 1 - Representação gráfica das causas de morte globais no ano de 2016. Fonte: WHO, 2018 - Noncomunicable diseases - Country profiles

Há uma clara relação entre morte prematura por DCNT e níveis de renda de um país. Em 2016, 78\% de todas as mortes por DCNT ocorreram em países de baixa ou média renda. Adultos desses países têm quase o dobro do risco de morrer de uma DCNT que adultos de países de alta renda (22\% contra $12 \%$, 
respectivamente) (1) .

A maior parte das mortes prematuras por DCNT são altamente preveníveis através de políticas que instrumentalizem os sistemas de saúde para responder de forma mais efetiva e equitativa às necessidades da população com DCNT e que influenciem de forma efetiva outros setores da sociedade para reduzir os fatores de risco em nível populacional, especialmente o tabagismo, os maus hábitos dietéticos, o sedentarismo e o uso nocivo do álcool.

\subsubsection{Doença cardiovascular no Brasil}

Dados do DATASUS(2) apuraram que houve 358.882 óbitos por doenças do aparelho circulatório em 2017, dos quais 115.058 foram por doença isquêmica do coração e 101.195 foram por doença cerebrovascular. Isso corresponde a $8,8 \%$ e $7,7 \%$ de todos os óbitos do período, respectivamente. Juntos, doença isquêmica do coração e cerebrovascular são responsáveis por 16,5\% de todos os óbitos em 2017.

\subsection{Aterosclerose}

A aterosclerose é fenômeno central na patogênese das doenças cardiovasculares, tais como doença isquêmica coronariana, aterosclerose aórtica resultando em aneurisma, acidente vascular cerebral (AVC) e 
insuficiência arterial periférica. É uma condição sistêmica caracterizada por lesões na camada íntima da artéria, denominadas ateromas ou placas ateromatosas, que invadem e obstruem o lúmen vascular e enfraquecem a camada média subjacente (3). A American Heart Association divide as lesões ateroscleróticas em seis tipos histológicos de gravidade progressivamente maior, desde células espumosas isoladas, estrias gordurosas, ateromas e fibroateromas, até as lesões avançadas. A disfunção endotelial, caracterizada pelo aumento da permeabilidade do endotélio às lipoproteínas pode iniciar a formação de placas de aterosclerose, seguida pela oxidação do colesterol-LDL, que desencadeia a reação inflamatória.

Em artérias pequenas, os ateromas podem obstruir os lúmens, comprometendo o fluxo sanguíneo dos órgãos mais distantes e resultar em isquemia. As placas podem se romper e precipitar formando trombos que aumentam a obstrução arterial. Em artérias de maior calibre, as placas invadem a média subjacente e enfraquecem as paredes do vaso, causando aneurismas que podem se romper. Ateromas extensos podem ser friáveis, liberando êmbolos para a circulação distal (3).

\subsection{Aterosclerose subclínica: a espessura íntima-média de artérias carótidas (EIMC)}


A EIMC é um marcador não invasivo de aterosclerose subclínica e pode ser utilizada para identificar indivíduos sob maior risco de doença arterial. Diversos estudos de coortes e meta-análises descreveram o uso da EIMC na predição de risco cardiovascular $(4,5,14-16,6-13)$. O grau de proliferação fibroblástica e migração de células de musculo liso da camada média para o espaço subendotelial de artérias pode ser inferido pela medida da EIMC. Essa medida tem sido usada em pesquisas como marcadora de aterosclerose subclínica e é um fator de risco independente para doença isquêmica do coração $(4-8,16)$.

$\mathrm{Na}$ coorte de descendentes do estudo de Framingham, encontrou-se associação entre a EIMC e história familiar de doença coronariana precoce e maiores níveis de proteína-C-reativa e interleucina-6 (4). O estudo Atherosclerosis Risk in Communities $(A R I C)$, uma grande coorte multicêntrica, concluiu que a adição da medida da EIMC e da presença de placas aos fatores de risco tradicionais aumentaram o poder de predição de risco para doença coronariana (5).

Duas meta-análises estudaram a associação entre EIMC de artérias carótidas por ultrassom e predição de risco cardiovascular $(6,7)$. Na primeira (6), que incluiu 36.984 participantes, a associação entre o aumento da EIMC, verificado através de duas medidas com intervalo de tempo médio de quatro anos, e a predição de risco cardiovascular na população geral permaneceu controversa. Outra meta-análise de 2013 concluiu que há associação entre a 
EIMC e infarto do miocárdio ("Hazard Ratio" = 1,26, intervalo de confiança de $95 \%$ [IC 95\%] $=1,20-1,31$ ) e AVC ("Hazard Ratio" $=1,15$, IC 95\% = 1,261,36), apesar da adição desse teste nos modelos atuais de predição de risco não resultar em aumento da performance desses modelos (7).

\subsection{Estratégias de prevenção populacionais e estudos de vizinhança}

Geoffrey Rose, em seu artigo "Sick individuals and sick populations" (17), destacou as vantagens do que chamou de "Estratégia populacional" no que diz respeito a estratégias de prevenção primária em saúde. A estratégia populacional, segundo Rose, visa controlar os determinantes da incidência das doenças, diminuir a média dos seus valores e deslocar a distribuição de uma exposição em direção a um nível mais favorável de risco. Tal estratégia tem o potencial de remover verdadeiramente as causas das doenças e atinge positivamente toda a população (e não só os indivíduos classificados como de alto risco). Dessa forma, ela contrasta com as estratégias preventivas "de alto risco", cujos esforços levam a resultados paliativos, com pouco benefício para populações, uma vez que, frequentemente, convida indivíduos a mudanças de hábitos e estilo de vida em um contexto de normas sociais contrárias e que devem ser repetidas geração após geração (Figura 2). 


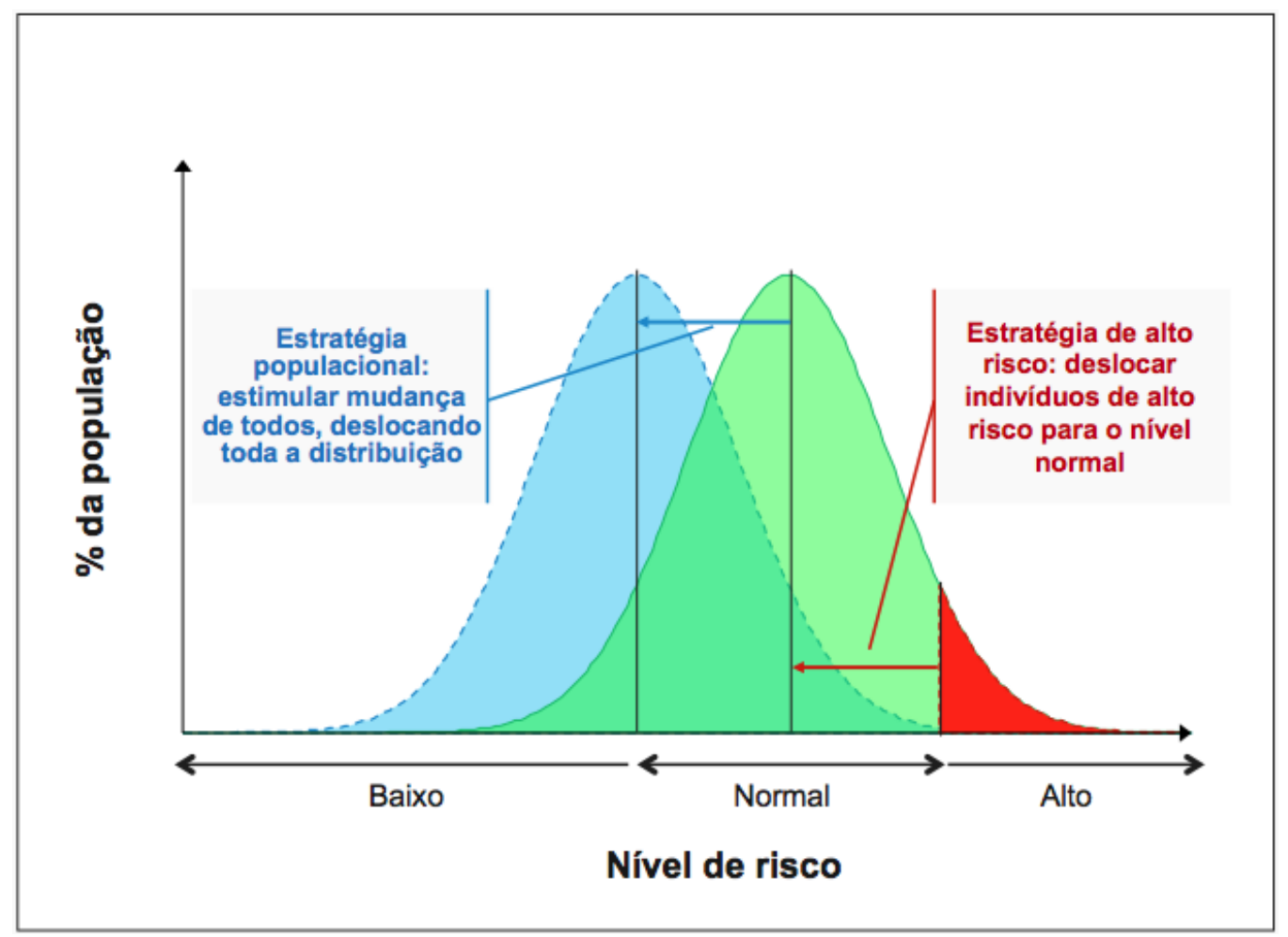

Figura 2 - Estratégias de prevenção populacional e de alto risco, segundo Rose. Deslocar toda a população em direção a um nível de menor risco beneficia mais indivíduos do que atuar somente tentando trazer os indivíduos de alto risco para um nível de risco mais baixo.

Fonte: adaptado de Rose, 2001 (17)

No que diz respeito à identificação de fatores de risco cardiovasculares e cerebrovasculares, a literatura médica já tem publicada extensa quantidade de em nível individual. No entanto, este paradigma que dá ênfase aos hábitos do indivíduo falha em considerar fatores de risco contextuais, relacionados ao ambiente de vida e que podem influenciar comunidades, vizinhanças e bairros atingindo populações inteiras. 


\subsection{Estudos de vizinhança}

Os estudos de vizinhança em saúde são um campo de estudo bem estabelecido em epidemiologia social (18). Muitos tipos de estudos têm sido conduzidos, incluindo estudos ecológicos relacionando características da área com morbidade e mortalidade, análises multinível e análises comparando pequenos números de vizinhanças bem definidas. Vários fatores estimularam o surgimento do interesse em investigar o papel da vizinhança no risco à saúde, incluindo o reconhecimento de que os determinantes sociais das doenças operam através de processos muito diversos. Estudos de vizinhança postulam que efeitos contextuais podem estar relacionados à saúde de forma independente das variáveis individuais.

\subsubsection{Estudos de vizinhança, DCV e aterosclerose subclínica}

As doenças cardiovasculares e seus fatores de risco também têm sido alvo de pesquisas que investigam a sua relação com características da vizinhança (19). Vizinhanças não são o único contexto ambiental potencialmente relevante ao desenvolvimento e prognóstico de doenças cardiovasculares. Os ambientes laboral, familiar e escolar são todos muito importantes. Fatores em nível mais macrossociais, tais como o consumo de alimentos processados, a dependência 
de automóvel para o transporte, a propaganda de cigarros e bebidas adoçadas e a qualidade da legislação e regulações para a comercialização de produtos contendo tabaco também têm papel central. No entanto, a utilização de medidas em nível de vizinhança são importantes por pelo menos três razões. Primeiramente, boa parte desses fatores macrossociais se expressam e agem sobre os indivíduos através dos ambientes físicos e sociais em que as pessoas moram. Ou seja, o estudo das vizinhanças permite entender os processos que ligam esses grandes fatores sociais e econômicos ao aparecimento de DCV de maneira muito concreta. Em segundo lugar, as grandes segregações sociais observadas no Brasil (e em muitos outros países) sugerem que essas diferenças entre as vizinhanças podem ser importantes contribuintes das disparidades em DCV. E, por fim, as diferenças entre as vizinhanças não são naturais, mas sim resultados do impacto de políticas públicas ou da ausência destas e são, portanto, diretamente passíveis de intervenção.

A forma como o ambiente de vizinhanças pode influenciar no risco e desenvolvimento de DCV tem sido pensada por pesquisadores através de um esquema conceitual reproduzido na Figura 3. 


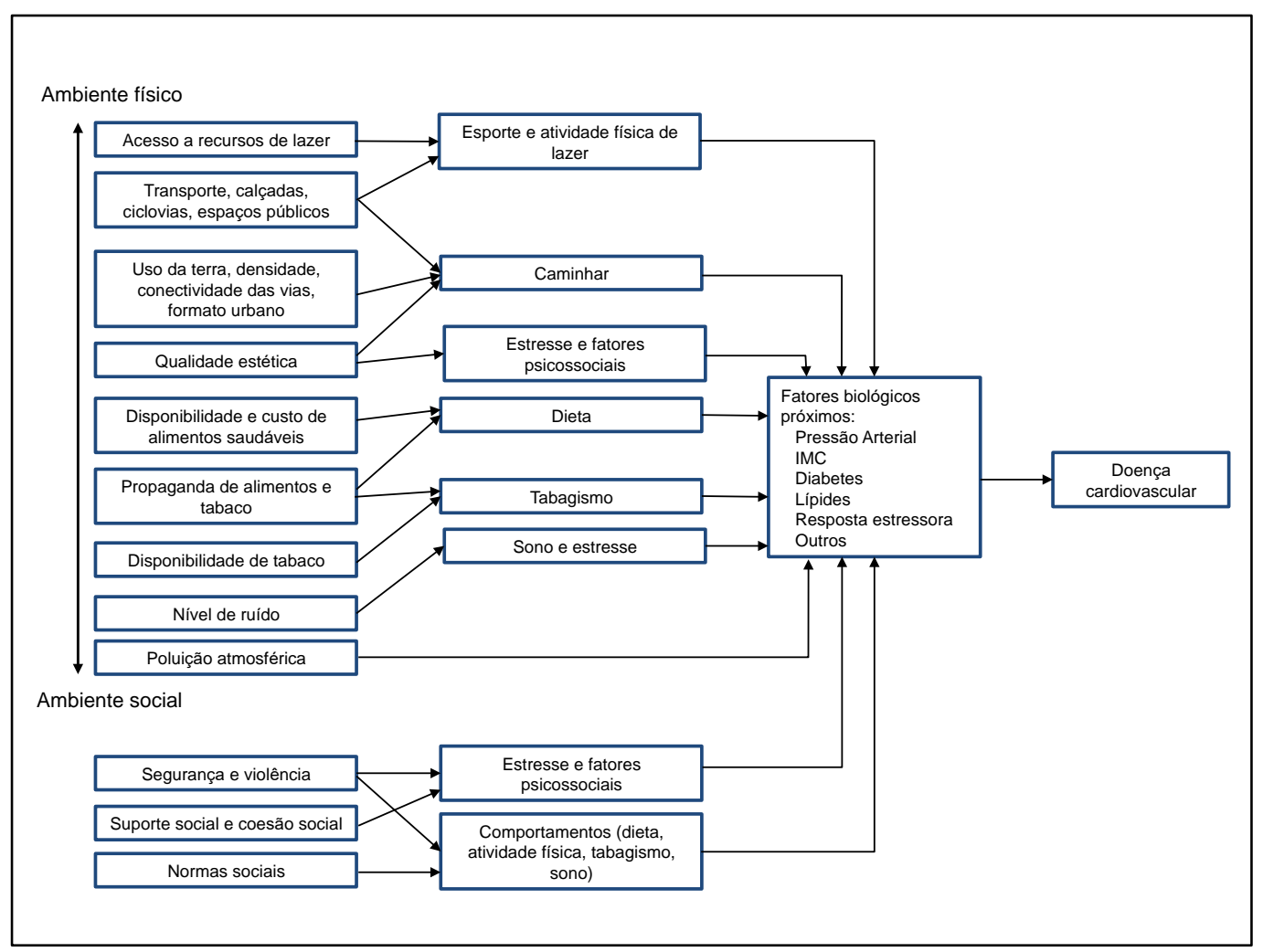

Figura 3 - Esquema conceitual das relações hipotéticas entre ambientes de vizinhança e DCV.

Fonte: adaptado de Diez-Roux, 2016(19)

\section{Características dos estudos de vizinhança e DCV}

Uma revisão da literatura publicado em 2009 mostrou que 35 dos 40 estudos até aquela data eram de apenas três países: Reino Unido, Suécia e Estados Unidos da América, e que apenas quatro de 40 estudos avaliaram DCV subclínica e seus resultados não são totalmente concordantes (20-24).

O primeiro estudo que avaliou a associação entre DCV subclínica e características de vizinhança foi conduzido em 2004 e incluiu 3.545 idosos. Uma escala composta por seis variáveis a respeito de renda, riqueza, 
educação e ocupação foi utilizada para avaliar características da vizinhança dos participantes. DCV subclínica foi avaliada de diversas maneiras, incluindo EIMC. Sessenta por cento dos participantes tinham algum indicador de doença subclínica, que se mostrou inversamente associada às características de vizinhança após ajuste para indicadores individuais, mas essa associação não se manteve significante após ajuste para fatores socioeconômicos individuais (21).

O estudo $A R I C$ investigou a associação entre aterosclerose subclínica medida por EIMC e condição socioeconômica em nível individual e de vizinhança. Foram obtidas informações individuais e de vizinhança para nível socioeconômico por georeferenciamento. Encontrou-se relação entre aterosclerose subclínica e baixo nível socioeconômico individual. No entanto, quando foram considerados fatores individuais e de vizinhança simultaneamente, a associação permaneceu apenas para nível individual (20).

Em Pitsburgo, Pensilvânia, um estudo realizado em 2006 entre homens hipertensos sem tratamento, revelou que aqueles que viviam em comunidades pobres definidas por níveis de renda, educação, pobreza, desemprego e custos de moradia pelo censo nacional apresentavam maior risco de apresentar aterosclerose subclínica, quando comparados com residentes de áreas mais $\operatorname{ricas}(22)$.

Outro estudo de corte realizado em uma amostra de 4.033 indivíduos da população geral do estudo Malmö Diet and Cancer, na cidade de Mälmo, 
Suécia, em 2007, encontrou lesões ateroscleróticas subclínicas mais extensas em artérias carótidas em pessoas que vivem em áreas mais desfavorecidas, definidas por áreas com mais desemprego, dependência de ajuda governamental, porcentagem de pessoas com apenas cidadania estrangeira entre os estrangeiros, taxas de migração, que persistiu mesmo após ajuste para indicadores individuais(23).

Em 2010, Murray et al. utilizaram dados do Multi-Ethnic Study of Atherosclerosis (MESA) para determinar grupos de trajetórias de pobreza em nível de vizinhança ao longo de 20 anos e correlacionar com aterosclerose subclínica, medida por EIMC, usando modelos de trajetória de classe latente. Os autores classificaram os participantes em seis grupos segundo o tipo de trajetória que haviam seguido no que diz respeito à pobreza das vizinhanças pelas quais passaram. Maiores níveis de pobreza na vizinhança acumulada ao longo da vida estiveram geralmente associados com piores desfechos cardiovasculares, principalmente entre as mulheres, em modelos ajustados por idade. Após ajuste para posição socioeconômica, raça e etnia, os intervalos de confiança ficaram muito grandes e as tendências de associação perderam a significância(25).

A mesma coorte publicou, em 2016, resultados de 5.950 participantes acompanhados durante 12 anos verificando a associação entre características da vizinhança, tais como disponibilidade de alimento saudável, ambiente para atividade física, ambiente de lazer e social e aterosclerose subclínica medida 
por escore de cálcio coronariano. Apenas o acesso a recursos de alimento saudável na vizinhança estiveram associados ao desenvolvimento mais lento de aterosclerose subclínica naquela amostra (26).

Em 2017, um estudo mexicano avaliou a associação entre exposição à violência e DCV subclínica em mulheres (27). Quarenta por cento das 634 mulheres sem DCV clínica reportaram terem sofrido violência sexual ou física no passado. Em relação a mulheres não expostas à violência, as expostas apresentaram maiores EIMC. A associação se mostrou mais forte quando a violência fora praticada por estranhos, especialmente roubo ou assalto. Apesar de não ter avaliado diretamente as características de vizinhança, a exposição à violência por estranhos encontrada no estudo deve ter ocorrido nos locais pelos quais essas mulheres mais transitam, principalmente o entorno de suas residências.

É preciso destacar que a maior parte dos estudos sobre a relação entre aterosclerose subclínica e vizinhança foram realizados em países ricos(20$22,24,25,28)$.

\section{Diferenças entre os sexos}

Alguns dos estudos encontraram resultados que indicam que características da vizinhança possam ter influências diferentes entre homens e 
mulheres, com maior influência da vizinhança em fatores de risco cardiovasculares em mulheres do que em homens(25,29-31).

Em sua publicação de trajetórias de vizinhança com a população do estudo MESA, Murray et. al. encontrou maior risco cardiovascular em mulheres mais expostas a vizinhança pobre, mas não em homens (25).

Grimaud et. al. publicou um estudo em 2013 explorando particularmente as diferenças entre os gêneros na associação entre status socioeconômico e aterosclerose subclínica. Status socioeconômico da vizinhança esteve associado inversamente à EIMC em mulheres apenas(29) .

Lemelin et. al. também descreveu evidências de heterogeneidade de efeitos de posição socioeconômica em nível de vizinhança por gênero na população do MESA em 2009. Eles encontraram associação entre posição socioeconômica da vizinhança com menores EIMC entre mulheres(30).

Em sua população de adultos mais jovens, o CARDIA também encontrou associação entre aterosclerose subclínica, medida por escore de cálcio de artérias coronárias, e pobreza em nível de vizinhança e coesão social apenas entre mulheres(31). Maior relação das mulheres com a comunidade e exposição a fatores da vizinhança podem explicar essa diferença.

\section{Medidas de vizinhança por questionários}


Questionários podem ser utilizados para acessar a percepção de cada indivíduo de sua vizinhança. Uma série de escalas têm sido utilizadas em estudos para capturar construtos de vizinhança, incluindo: qualidade estética, caminhabilidade/ambiente para atividade física, disponibilidade de alimento saudável, segurança, crime violento, coesão social, atividades com vizinhos, problemas de vizinhança e desordem física e social (32-38).

A confiabilidade das medidas auto-referidas de vizinhança com possível impacto em doenças cardiovasculares e seus fatores de risco foi verificada em um estudo nova-iorquino. Pesquisadores aplicaram os questionários através de contato telefônico e encontros presenciais. As questões foram repetidas duas semanas depois. O coeficiente de Cronbach's alfa variou entre 0,77 e 0,94 e correlação teste-reteste variou entre 0,73 e 0,91. Para quatro dos seis domínios avaliados, o teste-reteste foi superior a $0.80(39)$.

São necessários mais estudos em países pobres para investigar o papel das características da vizinhança como fator de risco para DCV, onde as desigualdades sociais são mais acentuadas. Além disso, a doença aterosclerótica subclínica foi pouco estudada em estudos de vizinhança $(25,26,30)$. O acesso ao risco em fase pré-clínica e a gradação do risco cardiovascular conforme a progressão da lesão permitem entender melhor fatores de risco iniciais para a doença aterosclerótica, onde provavelmente será possível delinear programas de prevenção mais efetivos para ser implementados ao nível populacional. Por fim, há lacunas no entendimento do 
papel que a vizinhança pode ter entre homens e mulheres no processo de aterosclerose. 
2. Objetivos 
- Investigar a associação entre a percepção sobre características de vizinhança e aterosclerose subclínica.

\subsection{Objetivos específicos:}

- Investigar a associação entre percepção de coesão social na vizinhança e EIMC

- Investigar a associação entre percepção de ambiente para atividade física na vizinhança e EIMC

- Investigar a associação entre percepção de disponibilidade de alimento saudável na vizinhança e EIMC

- Investigar a associação entre percepção de segurança na vizinhança e EIMC

- Investigar a associação entre percepção de violência na vizinhança e EIMC

- Investigar a associação entre percepção de vitimização pessoal na vizinhança e EIMC

\subsection{Hipótese}

Há maior prevalência de aterosclerose subclínica entre indivíduos que têm piores percepções sobre as características de sua vizinhança. 
3. Métodos 


\subsection{Desenho do estudo}

Trata-se de um estudo observacional transversal.

\subsection{Casuística}

O ELSA-Brasil é uma coorte prospectiva multicêntrica cujo objetivo é investigar aspectos epidemiológicos, clínicos e moleculares das doenças crônicas, principalmente as doenças cardiovasculares e o diabetes tipo 2. Os participantes são adultos com idade entre 35 e 74 anos no momento da inclusão na coorte, o que ocorreu entre 2008 e 2010, e são funcionários ativos ou aposentados de instituições públicas de ensino superior. Seis instituições participam do ELSA-Brasil: as universidades federais da Bahia, de Minas Gerais, do Espírito Santo e do Rio Grande do Sul, além da Fundação Oswaldo Cruz no Rio de Janeiro e do Hospital Universitário da Universidade de São Paulo (HU-USP). Todos os participantes preencheram e assinaram o termo de consentimento livre e esclarecido no momento da sua inclusão no estudo.

15.105 participantes se inscreveram na linha de base do estudo, quando foram obtidas informações sociodemográficas, históricos médico, ocupacional e familiar, de saúde mental, estilo de vida e acesso a serviços de saúde. $\mathrm{Na}$ mesma etapa, foram realizadas medidas antropométricas, da pressão arterial, colhidos exames laboratoriais (como por exemplo, glicemia, teste de tolerância 
oral à glicose, lipídeos, creatinina, marcadores inflamatórios, microalbuminúria) e realizados exames de imagem (como por exemplo, espessura íntima-média de artérias carótidas, velocidade de onda de pulso e ecocardiografia). $(40,41)$

\subsubsection{Critério de inclusão}

Ser funcionário ativo ou aposentado das instituições participantes e ter idade entre 35 e 74 anos. 15.105 participantes atenderam aos critérios de inclusão na linha de base do ELSA-Brasil.

\subsubsection{Critérios de exclusão}

\section{Critérios de exclusão do ELSA-Brasil}

- Gestação atual ou recente (até 4 meses antes da primeira entrevista)

- Intenção de sair do trabalho na instituição em futuro próximo

- Déficit cognitivo ou de comunicação severo

- Se aposentado, residir fora da área metropolitana correspondente ao centro de estudo 
- Sujeitos com história clínica de aterosclerose manifesta, tais como doença arterial coronariana, acidente vascular cerebral, insuficiência arterial periférica ou com antecedente de revascularização do miocárdio;

- Sujeitos com informações incompletas no questionário de vizinhança;

- Sujeitos com informações incompletas das covariáveis;

- Sujeitos que não realizaram o exame de EIMC ou dos quais o exame não apresentou qualidade satisfatória;

\subsection{Variável preditora: questionário da percepção sobre características de vizinhança}

No ELSA-Brasil, a percepção dos participantes acerca das características de sua vizinhança foi acessada através da aplicação de um questionário autoreferido adaptado e validado para a realidade brasileira (Anexos, Quadro 1). 0 estudo que verificou a confiabilidade da adaptação mostrou boa reprodutibilidade e consistência. (Chronbach's alfa entre 0,60 e 0,84) (42).

No início do bloco de questões, os entrevistados foram orientados a pensar sua vizinhança como: "a área geral do entorno da residência onde o/a senhor/senhora costuma realizar atividades de rotina como, por exemplo, fazer compras, ir ao parque ou visitar vizinhos".

As perguntas captaram a percepção dos participantes a respeito de características psicossociais e físicas do ambiente em que viviam agrupadas 
em seis domínios: (1) coesão social, (2) ambiente para atividade física, (3) disponibilidade de alimento saudável, (4) segurança, (5) violência percebida e (6) vitimização pessoal. Cada domínio possuía um número variável de perguntas e as opções de resposta também variaram em diferentes domínios, conforme abaixo descrito.

Antes de responder às perguntas dos quatro primeiros domínios (coesão social, ambiente para atividade física, disponibilidade de alimento saudável e segurança), o entrevistado foi orientado a escolher a melhor resposta dentre as opções de uma escala Likert: 1 - "concordo totalmente", 2 - "concordo parcialmente", 3 - "não concordo nem discordo", 4 - "discordo parcialmente", ou 5 - "discordo totalmente", apresentadas em um cartão, em relação a cada item.

Em seguida, antes de responder às perguntas sobre o domínio intitulado "violência percebida", o entrevistado foi orientado a responder "com que frequência os fatos descritos haviam ocorrido nos últimos seis meses", de acordo com o que ele(a) sabia sobre a sua vizinhança, e não somente com o que tinha presenciado ou sofrido", escolhendo a melhor resposta dentre as opções: 1 - "frequentemente", 2 - "às vezes", 3 - "raramente", ou 4 - "nunca".

Por fim, o entrevistado era questionado sobre o tempo de moradia na sua vizinhança e sobre vitimização pessoal, única pergunta em que as respostas possíveis eram apenas "sim" ou "não". 
Para análise, foi necessário codificar reversamente algumas delas para que a melhor pontuação sempre representasse as melhores percepções de vizinhança.

\subsection{Variável de desfecho: espessura íntima-média de carótidas}

A EIMC foi acessada em todos os sujeitos do ELSA Brasil de forma padronizada, utilizando o aparelho de ultrassom Aplio XG ${ }^{T M}$ da Toshiba, com transdutor linear de $7,5 \mathrm{MHz}$. Após o exame da bifurcação carotídea para verificar a presença de placas (medida não utilizada no presente estudo), realizaram-se as medidas da espessura das artérias carótidas comuns. Imagens foram adquiridas por uma extensão de $1 \mathrm{~cm}$, iniciando $1 \mathrm{~cm}$ abaixo da bifurcação carotídea. Depois de gravadas, foram enviadas ao laboratório central de imagem de ultrassom em São Paulo, onde as imagens de três ciclos cardíacos foram analisadas utilizando um programa de computador automatizado $\left(\mathrm{MIA}^{\mathrm{TM}}\right)$. Foram consideradas válidas as imagens que visualizaram com clareza o reparo anatômico das artérias carótidas comuns, as interfaces entre o lúmen do vaso e a parede externa e as interfaces entre a média e a adventícia da parede externa do vaso. A EIMC foi calculada

utilizando a média das medidas obtidas das artérias carótidas esquerda e direita $(40,43)$.

\subsection{Variáveis de confusão}


Foram incluídas na análise possíveis variáveis de confusão entre a percepção das características de vizinhança e a aterosclerose subclínica, incluindo: variáveis sociodemográficas e de risco cardiovascular descritas abaixo:

Sociodemográficas:

- Idade;

- Sexo;

- Educação (ensino médio ou menos e ensino superior);

- Cor (branco, negro, pardo e outros) e

- Renda (categorizada em tercis)

Fatores de risco cardiovasculares:

- Hipertensão arterial sistêmica (definida por diagnóstico médico prévio, pressão arterial sistólica $\geq 140 \mathrm{mmHg}$, pressão arterial diastólica $\geq$ 90mmHg ou uso de medicação anti-hipertensiva);

- Diabetes mellitus (definida por diagnóstico médico prévio, glicemia de jejum sérica $\geq 126 \mathrm{mg} / \mathrm{dL}$, teste de tolerância oral à glicose de $75 \mathrm{~g} \geq$ $200 \mathrm{mg} / \mathrm{dL}$ após 2 horas ou hemoglobina glicada $\geq 6,5 \%$; uso de medicação hipoglicemiante oral ou insulina);

- Nível de colesterol de baixa densidade (colesterol-LDL);

- Nível de colesterol de alta densidade (colesterol-HDL); 
- Índice de massa corpórea (IMC) (calculado dividindo o peso do participante em quilogramas pela altura ao quadrado em metros);

- Nível de atividade física de lazer avaliada pelo Questionário internacional de atividade física (IPAQ) e categorizada em leve, moderada ou vigorosa;

- Tabagismo (categorizado em não tabagista, tabagista prévio ou atual);

- Abuso de álcool (definido como consumo maior que $210 \mathrm{~g}$ por semana para homens e maior que $140 \mathrm{~g}$ por semana para mulheres).

Além das variáveis de confusão acima descritas, ajustamos a análise pelo tempo em anos de residência no endereço atual.

\subsection{Análise estatística}

A EIMC foi utilizada como variável contínua. A pontuação em cada domínio do questionário de percepção de características de vizinhança foi considerada a variável preditora. Modelos de regressão linear hierarquicamente ajustados descreveram a associação entre a pontuação em cada domínio e a EIMC.

Após a análise crua, o modelo de regressão linear foi ajustado para idade, sexo e anos de residência. Em segundo lugar, foram incluídos ajustes por renda, educação e cor da pele. Por último, adicionaram-se os fatores de risco cardiovasculares ao modelo, a saber: hipertensão, diabetes, dislipidemia, 
tabagismo, abuso de álcool, nível de atividade física e índice de massa corpórea (IMC).

Também foi realizada uma análise estratificada por anos de residência, para a qual foi definido o corte de 5 anos, que corresponde ao $25^{\circ}$ percentil da distribuição desta variável.

Adicionalmente, foram testadas interações entre sexo e cada domínio de vizinhança, baseados em evidência prévia de que essa associação poderia ser diferente entre homens e mulheres(25,30,31).

Finalmente, para minimizar o potencial impacto de dados faltantes para EIMC, foi utilizado o recurso estatístico de imputação múltipla, capaz de produzir medidas mais acuradas num contexto de muitos dados faltantes em comparação com outros métodos (44). Para isso, foi utilizado o comando mi no Stata 15 (Stata Corp LP, College Station, TX). Todos os testes foram bicaudais e o nível alfa foi definido em $5 \%$. 
4. Resultados 


\subsection{Casuística}

Entre os 15.105 participantes avaliados na linha de base do ELSA-Brasil, 9.923 tinham critérios de elegibilidade para este estudo, resultando no fluxograma apresentado na figura 4.

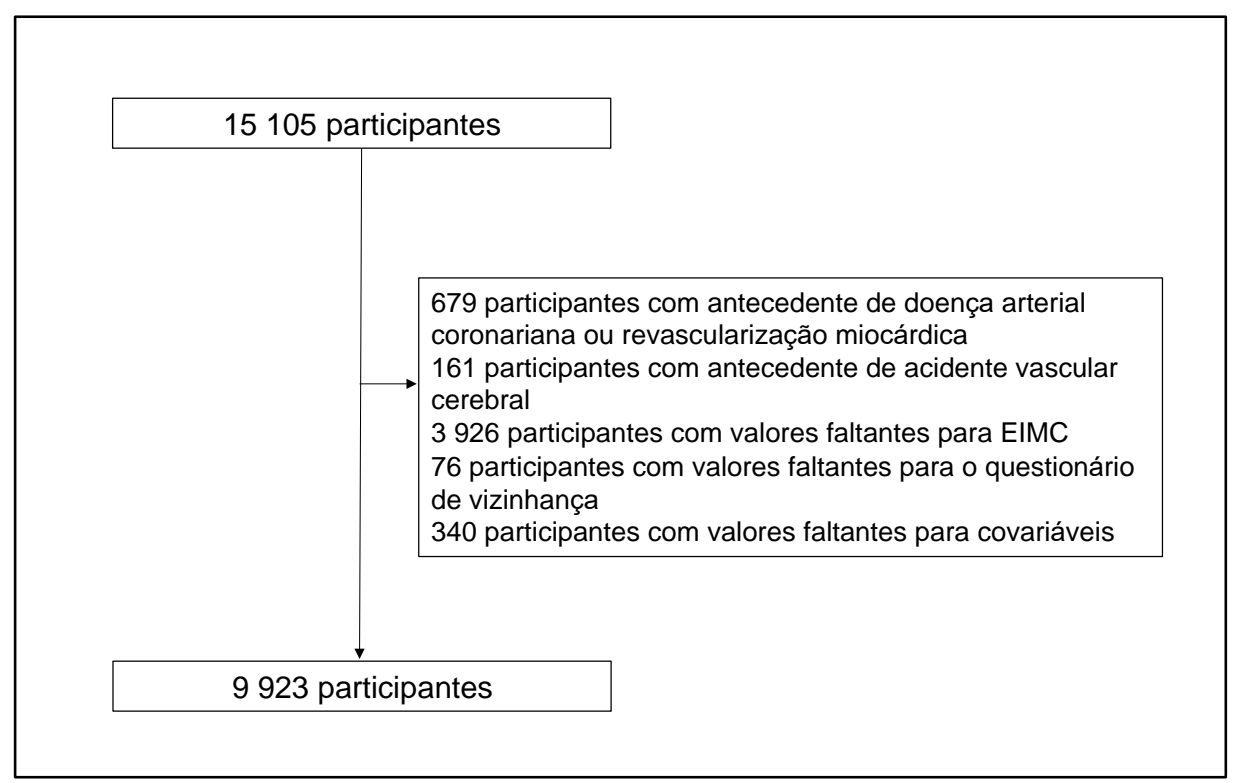

Figura 4 - Fluxograma dos participantes

\subsection{Análise descritiva}

A idade média da amostra foi de 51,5 anos (desvio-padrão $=8,9$ anos). Foi observado predomínio do sexo feminino (56\%), da cor branca (55\%) e de indivíduos com pelo menos o ensino superior completo (53\%). 
Com relação à presença de fatores de risco cardiovasculares, 32\% dos participantes foram classificados como hipertensos, $18 \%$ diabéticos, $6,9 \%$ alcoolistas abusivos, 13,2\% eram tabagistas atuais e $29,3 \%$ relatavam serem ex-tabagistas. Houve predomínio de pessoas sedentárias na amostra (77\%). A média de colesterol-LDL da população foi de 131,3 $\pm 34,2 \mathrm{mg} / \mathrm{dl}$ e do índice de massa corpórea foi de $26,8 \pm 4,6 \mathrm{~kg} / \mathrm{m}^{2}(1)$. 
Tabela 1 - Associação entre EIMC $(\mu \mathrm{m})$ e variáveis demográficas e clínicas, ajustado por idade e sexo $(n=9.923)$

\begin{tabular}{|c|c|c|}
\hline VARIÁVEL & Média (DP) ou \% & $\beta$ (intervalo de confiança de $95 \%)$ \\
\hline Idade (anos) & $51,5(8,9)$ & $8,54(8,22 ; 8,86)$ \\
\hline Sexo & 44 & $45,79(39,34 ; 52,24)$ \\
\hline \multicolumn{3}{|l|}{ Cor } \\
\hline Branca & 55,8 & 0 (referência) \\
\hline Parda & 25,4 & $17,17(10,42 ; 23,91)$ \\
\hline Preta & 15,1 & $50,81(42,66 ; 58,97)$ \\
\hline Outras & 3,8 & $7,75(-7,12 ; 22,62)$ \\
\hline \multicolumn{3}{|l|}{ Educação } \\
\hline $\begin{array}{l}\text { Ensino médio ou } \\
\text { menos }\end{array}$ & 47,5 & 0 (referência) \\
\hline $\begin{array}{l}\text { Ensino superior ou } \\
\text { mais }\end{array}$ & 53,5 & $-34,10(-39,74 ;-28,47)$ \\
\hline \multicolumn{3}{|l|}{ Renda mensal $(\mathrm{R} \$)$} \\
\hline$<968,2$ & 37 & 0 (referência) \\
\hline $968,2-1.971$ & 33,7 & $-20,23(-26,94 ;-13,51)$ \\
\hline$>1.971$ & 29,3 & $-31,27(-38,39 ;-24,52)$ \\
\hline Hipertensão & 32 & $73,19(66,00 ; 79,38)$ \\
\hline Diabetes & 18 & $54,32(46,84 ; 61,80)$ \\
\hline \multicolumn{3}{|l|}{ Dislipidemia } \\
\hline LDL & $131,3(34,2)$ & $0,42(0,34 ; 0,50)$ \\
\hline HDL & $57,2(14,7)$ & $-1,32(-1,53 ;-1,12)$ \\
\hline Abuso de álcool ${ }^{1}$ & 6,9 & $16,05(4,76 ; 27,33)$ \\
\hline \multicolumn{3}{|l|}{ Tabagismo } \\
\hline Nunca & 57,5 & 0 (referência) \\
\hline Prévio & 29,3 & $16,88(10,34 ; 23,41)$ \\
\hline Atual & 13,2 & $30,99(22,36 ; 39,61)$ \\
\hline Atividade física & 23,3 & $-14,60(-22,31 ;-8,89)$ \\
\hline $\begin{array}{l}\text { Índice de massa } \\
\text { corpórea (kg/m2) }\end{array}$ & $26,8(4,6)$ & $8,34(7,75 ; 8,94)$ \\
\hline
\end{tabular}

Idade, sexo masculino, cor da pele negra ou parda tiveram associação com maiores valores de EIMC. Enquanto participantes com pelo menos ensino superior completo e maiores estratos de renda tiveram associação com 
menores valores de EIMC. Hipertensão, diabetes, nível de colesterol-LDL, abuso de álcool, tabagismo atual ou prévio e índice de massa corpórea estiveram associados com maiores EIMC, enquanto que maiores níveis de colesterol-HDL e atividade física mostraram associação com valores menores de EIMC (Tabela 1).

A pontuação dos participantes no questionário de vizinhança é descrito na Tabela 2 e os histogramas da pontuação em cada domínio, na figura 5. Médias e desvios padrões foram apresentados para aqueles domínios em que a distribuição da pontuação dos participantes mostrou-se normal (coesão social, ambiente para atividade física, disponibilidade de alimento saudável e segurança). Mediana e intervalo interquartílico foram utilizados para descrever a pontuação do domínio em que as respostas não tiveram distribuição normal (violência percebida). Para o domínio "vitimização pessoal”, de pergunta única

e resposta binária, apresentou-se a porcentagem de respostas. Conforme explicado na seção de métodos, as pontuações de algumas perguntas precisaram ser codificadas reversamente, para que as maiores pontuações representassem melhores percepções das características de vizinhança. 
Tabela 2 - Percepção de características de vizinhança $(n=9.923)$

\begin{tabular}{|c|c|c|c|c|}
\hline $\begin{array}{l}\text { DOMIINIO DE } \\
\text { CARACTERÍSTICAS } \\
\text { DE VIZINHANÇA }\end{array}$ & $\begin{array}{l}\text { Número de } \\
\text { perguntas do } \\
\text { domínio }\end{array}$ & $\begin{array}{l}\text { Tipos de respostas } \\
\text { possíveis }\end{array}$ & $\begin{array}{l}\text { Variação de } \\
\text { pontuação } \\
\text { possível }\end{array}$ & $\begin{array}{l}\text { Média (DP), } \\
\text { mediana ou \% }\end{array}$ \\
\hline Coesão social & 5 & Likert $1-5$ & $5-25$ & $17,2(3,6)$ \\
\hline $\begin{array}{l}\text { Ambiente para atividade } \\
\text { física }\end{array}$ & 9 & Likert 1 - 5 & $9-45$ & $32,9(7,3)$ \\
\hline $\begin{array}{l}\text { Disponibilidade de } \\
\text { alimento saudável }\end{array}$ & 4 & Likert 1 - 5 & $4-20$ & $15(2,7)$ \\
\hline Segurança & 3 & Likert 1 - 5 & $3-15$ & $9,5(3,1)$ \\
\hline Violência percebida & 5 & $\begin{array}{l}\text { Escala de } \\
\text { frequência } 1 \text { - } 4\end{array}$ & $5-20$ & $17(15 ; 19)^{1}$ \\
\hline Vitimização pessoal & 1 & Binária & Sim/Não & 67,7 \\
\hline
\end{tabular}

${ }^{1}$ Distribuição não normal. Média e intervalo interquartil. 


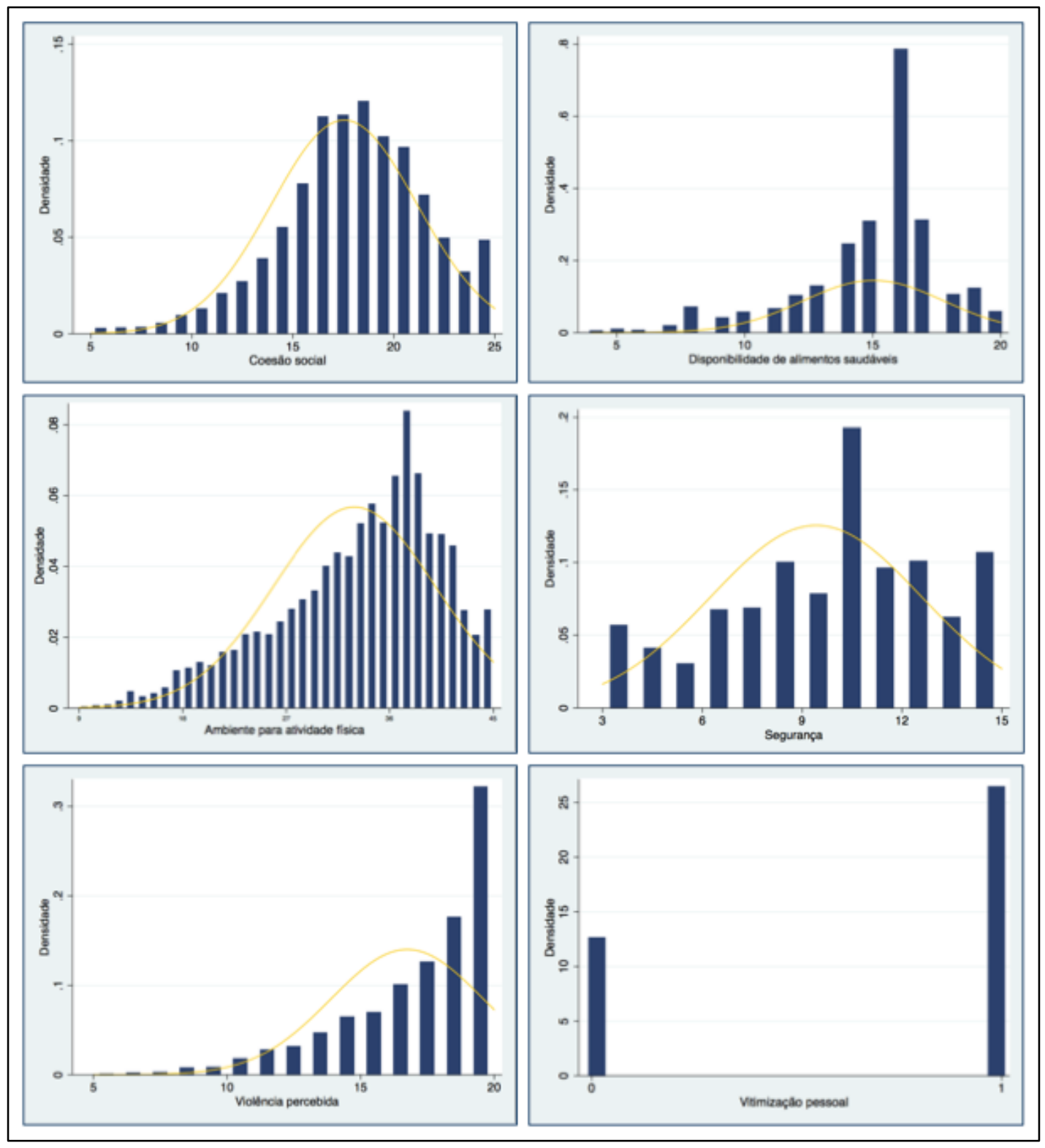

Figura 5 - Histograma da pontuação dos participantes em cada domínio do questionário. "Vitimização pessoal" é o único domínio composto por pergunta única de resposta binária $(0=\operatorname{sim} ; 1=$ não $)$

\subsection{Associação entre EIMC e percepção de vizinhança}

\subsubsection{Análise multivariada}


$\mathrm{Na}$ análise ajustada para idade, sexo e anos de residência, melhor percepção de ambiente para atividade física $(\beta=-0,62$, IC $95 \%=-1,01 ;-0,23$, $p=0,002)$ e menor violência percebida $(\beta=-1,95$, IC $95 \%=-2,96 ;-0,94, p<$ 0,001) mostraram associação com menor EIMC. (Tabela 3). Por outro lado, baixa percepção de vitimização pessoal esteve associada com maior EIMC. ( $\beta$ $=10,70$, IC $95 \%=4,55 ; 16.85, p=0,001)$. Quando cor e fatores socioeconômicos foram adicionados ao modelo, apenas baixa percepção de vitimização pessoal mostrou uma associação limítrofe com maior EIMC. ( $\beta$ = $6,12$, IC $95 \%=-0,01 ; 12,24, p=0,05)$. Quando o modelo foi ajustado para fatores de risco cardiovasculares, nenhuma associação foi encontrada entre percepção de características de vizinhança e EIMC 
Tabela 3 - Coeficientes e intervalos de confiança (IC) de 95\% para EIMC $(\mu \mathrm{m})$, de acordo com percepção de características de vizinhança $(n=9.923)$ - ELSA-Brasil - 2010

\begin{tabular}{|c|c|c|c|c|}
\hline $\begin{array}{c}\text { DOMÍNIO DE } \\
\text { CARACTERÍSTICAS } \\
\text { DE VIZINHANÇA }\end{array}$ & Crua & Modelo 1 & Modelo 2 & Modelo 3 \\
\hline & $\beta($ IC 95\%) & $\beta($ IC 95\%) & $\beta($ IC 95\%) & $\beta($ IC 95\%) \\
\hline Coesão social & $0,97(0,08 ; 1,86)$ & $-0,65(-1,44 ; 0,14)$ & $-0,09(-0,87 ; 0,70)$ & $0,17(-0,57 ; 0,91)$ \\
\hline $\begin{array}{l}\text { Ambiente para atividade } \\
\text { física }\end{array}$ & $0,28(-0,15 ; 0,72)$ & $-0,62(-1,01 ;-0,23)$ & $-0,26(-0.65 ; 0.13)$ & $-0,09(-0,46 ; 0,28)$ \\
\hline $\begin{array}{l}\text { Disponibilidade de } \\
\text { alimento saudável }\end{array}$ & $3,04(1,87 ; 4,21)$ & $0,27(-0.74 ; 1.32)$ & $0,62(-0,40 ; 1,64)$ & $0,66(-0,31 ; 1,62)$ \\
\hline Segurança & $1,80(0,78 ; 2,8)$ & $-0,13(-1,02 ; 0,76)$ & $-0,35(-1,23 ; 0,54)$ & $-0,62(-1,46 ; 0,22)$ \\
\hline Violência Percebida & $0,83(-0,31 ; 1,97)$ & $-1,95(-2,96 ;-0,94)$ & $-0,96(-1,97 ; 0,06)$ & $-0,54(-1,50 ; 0,42)$ \\
\hline Vitimização Pessoal & $8,36(1,46 ; 15,26)$ & $10,70(4,55 ; 16,85)$ & $6,12(-0,01 ; 12,24)$ & $3,02(-2,67 ; 8,71)$ \\
\hline
\end{tabular}

Modelo 1: Regressão linear simples, ajustada por idade, sexo e anos de residência

Modelo 2: Regressão linear simples, ajustada por idade, sexo, anos de residência, cor, renda e educação

Modelo 3: Regressão linear simples, ajustada por idade, sexo, anos de residência cor, renda, educação, hipertensão,

diabetes, dislipidemia, tabagismo, abuso de álcool, atividade física e IMC 


\subsubsection{Análise estratificada por anos de residência}

Entre participantes que moravam na vizinhança por mais de cinco anos, melhor percepção de ambiente para atividade física $(\beta=-1,00$, IC 95\% $=-1,46$; $-0,54, p<0,001)$ e baixa percepção de violência percebida ( $\beta=-1,80$, IC 95\% $=-2,98 ;-0,62, p=0,003)$ estiveram associados com menor EIMC, enquanto baixa percepção de vitimização pessoal mostrou uma associação com maiores EIMC $(\beta=11,08$, IC $95 \%=4,16 ; 17,99, p=0,002)$ na análise ajustada por idade e sexo (Tabela 4). Quando cor e variáveis socioeconômicas foram adicionados à análise, apenas melhor percepção de ambiente para atividade física mostrou associação com baixa EIMC entre aqueles participantes que viviam na vizinhança há mais de cinco anos $(\beta=-0,59$, IC $95 \%=-1,05 ;-0,12 p$ $=0,013)$. Entre os participantes que viviam na vizinhança por 5 anos ou menos, apenas baixa percepção de violência mostrou uma associação negativa com EIMC ( $\beta=-3,05$, IC 95\% $=-4,99 ;-1,12 p=0,002)$. Essa associação se manteve após ajuste para cor e fatores socioeconômicos $(\beta=-2,19$, IC $95 \%=-4,15$;$0,24, p=0,028)$. Nenhuma associação se manteve após a adição de ajuste para fatores de risco cardiovasculares entre participantes que viviam nos dois estratos de tempo de moradia na vizinhança. 
Tabela 4 - Coeficientes e intervalos de confiança (IC) de 95\% para EIMC $(\mu \mathrm{m})$ de acordo com percepção de características de vizinhança, estratificado por anos de residência $(n=9.923)$

\begin{tabular}{|c|c|c|c|}
\hline $\begin{array}{l}\text { DOMÍNIO DE } \\
\text { CARACTERÍSTICAS DE } \\
\text { VIZINHANÇA }\end{array}$ & Modelo 1 & Modelo 2 & Modelo 3 \\
\hline & $\beta$ (IC 95\%) & $\beta$ (IC 95\%) & $\beta$ (IC 95\%) \\
\hline \multicolumn{4}{|l|}{ Coesão social } \\
\hline 5 anos ou menos & $-0,65(-2,12 ; 0,83)$ & $-0,15(-1,63 ; 1,32)$ & $0,23(-1,18 ; 1,63)$ \\
\hline Mais de 5 anos & $-0,64(-1,57 ; 0,28)$ & $-0,04(-0,97 ; 0,88)$ & $0,14(-0,75 ; 1,01)$ \\
\hline \multicolumn{4}{|l|}{$\begin{array}{l}\text { Ambiente para atividade } \\
\text { física }\end{array}$} \\
\hline 5 anos ou menos & $0,24(-0,47 ; 0,95)$ & $0,57(-0,15 ; 1,28)$ & $0,76(0,07 ; 1,44)$ \\
\hline Mais de 5 anos & $-1,00(-1,46 ;-0,54)$ & $-0,59(-1,05 ;-0,12)$ & $-0,40(-0,84 ; 0,03)$ \\
\hline \multicolumn{4}{|l|}{$\begin{array}{l}\text { Disponibilidade de } \\
\text { alimento saudável }\end{array}$} \\
\hline 5 anos ou menos & $-0,67(-2,53 ; 1,19)$ & $-0,44(-2,28 ; 1,41)$ & $-0,18(-1,93 ; 1,58)$ \\
\hline Mais de 5 anos & $0,62(-0,61 ; 1,85)$ & $1,05(-0,17 ; 2,27)$ & $0,96(-0,19 ; 1,12)$ \\
\hline \multicolumn{4}{|l|}{ Segurança } \\
\hline 5 anos ou menos & $-0,56(-2,22 ; 1,10)$ & $-0,52(-2,17 ; 1,13)$ & $-0,89(-2,46 ; 0,67)$ \\
\hline Mais de 5 anos & $-0,03(-1,09 ; 1,02)$ & $-0,34(-1,39 ; 0,70)$ & $-0,57(-1,56 ; 0,42)$ \\
\hline \multicolumn{4}{|l|}{ Violência percebida } \\
\hline 5 anos ou menos & $-3,05(-4,99 ;-1,12)$ & $-2,19(-4,15 ;-0,24)$ & $-1,36(-3,23 ; 0,50)$ \\
\hline Mais de 5 anos & $-1,80(-2,98 ;-0,62)$ & $-0,66(-1,85 ; 0,52)$ & $-0,33(-1,45 ; 0,79)$ \\
\hline \multicolumn{4}{|l|}{ Vitimização pessoal } \\
\hline 5 anos ou menos & $-1,26(-15,11 ; 12,58)$ & $-1,57(-15,35 ; 12,21)$ & $-4,27(-17,38 ; 8,85)$ \\
\hline Mais de 5 anos & $11,08(4,16 ; 17,99)$ & $5,97(-0,92 ; 12,85)$ & $3.91(-2,60 ; 10,42)$ \\
\hline
\end{tabular}

Modelo 1: Regressão linear ajustada por idade e sexo

Modelo 2: Regressão linear ajustada por idade, sexo, cor, renda e educação

Modelo 3: Regressão linear ajustada por idade, sexo, cor, renda, educação, hipertensão, diabetes,

dislipidemia, tabagismo, abuso de álcool, atividade física e IMC 


\subsubsection{Análise de interação entre percepção de vizinhança e sexo}

Nós também incluímos termos de interação para cada domínio e sexo (Tabela 5). Foi encontrada uma associação limítrofe entre sexo e percepção de coesão social ( $p$-valor da interação $=0,06$ ) e entre sexo e percepção de segurança $(p=0,08)$. Melhor percepção de coesão social esteve associada com maior EIMC entre mulheres, enquanto melhor percepção de segurança esteve associada com menor EIMC entre homens. O termo de interação entre sexo e coesão social $(p=0,06)$ sugere uma associação mais forte entre mulheres. No entanto, nós provavelmente não tivemos força o suficiente para demonstrar que essa associação é significante entre mulheres na análise estratificada $(p=0,09)$.

Tabela 5 - Coeficientes e intervalos de confiança (IC) de 95\% para EIMC ( $\mu \mathrm{m})$ de acordo com percepção de características de vizinhança, estratificado por sexo $(n=9.923)$

\begin{tabular}{lccc}
\hline $\begin{array}{l}\text { DOMÍNIO DE } \\
\text { CARACTERIÍSTICAS } \\
\text { DE VIZINHANÇA }\end{array}$ & Homens & Mulheres & $\begin{array}{c}\text { P-valor da } \\
\text { interação }^{1}\end{array}$ \\
\hline $\begin{array}{l}\text { Coesão social } \\
\begin{array}{l}\text { Ambiente para atividade } \\
\text { física }\end{array}\end{array}$ & $-0,70(-1,95 ; 0,55)$ & $0,79(-0,11 ; 1,68)$ & 0,057 \\
$\begin{array}{l}\text { Disponibilidade de } \\
\text { alimento saudável }\end{array}$ & $-0,33(-2,00 ; 1,33)$ & $1,28(0,13 ; 2,43)$ & 0,179 \\
$\begin{array}{l}\text { Segurança } \\
\text { Violência percebida }\end{array}$ & $-1,57(-2,97 ;-0,16)$ & $0,14(-0,88 ; 1,15)$ & 0,076 \\
Vitimização pessoal & $-0,64(-2,24 ; 0,96)$ & $-0,48(-1,65 ; 0,69)$ & 0,844 \\
\hline
\end{tabular}

NOTA: Regressão linear ajustada por idade, cor, renda, educação, hipertensão, diabetes, dislipidemia, tabagismo, abuso de álcool, atividade física, IMC e anos de residência ${ }^{1} \mathrm{P}$-valor da interação entre cada domínio de características de vizinhança e sexo na associação entre cada domínio e a EIMC 


\subsubsection{Análise por método de imputação múltipla}

O método de imputação múltipla para dados faltantes sobre EIMC resultou no fluxograma de participantes apresentado na Figura 6.

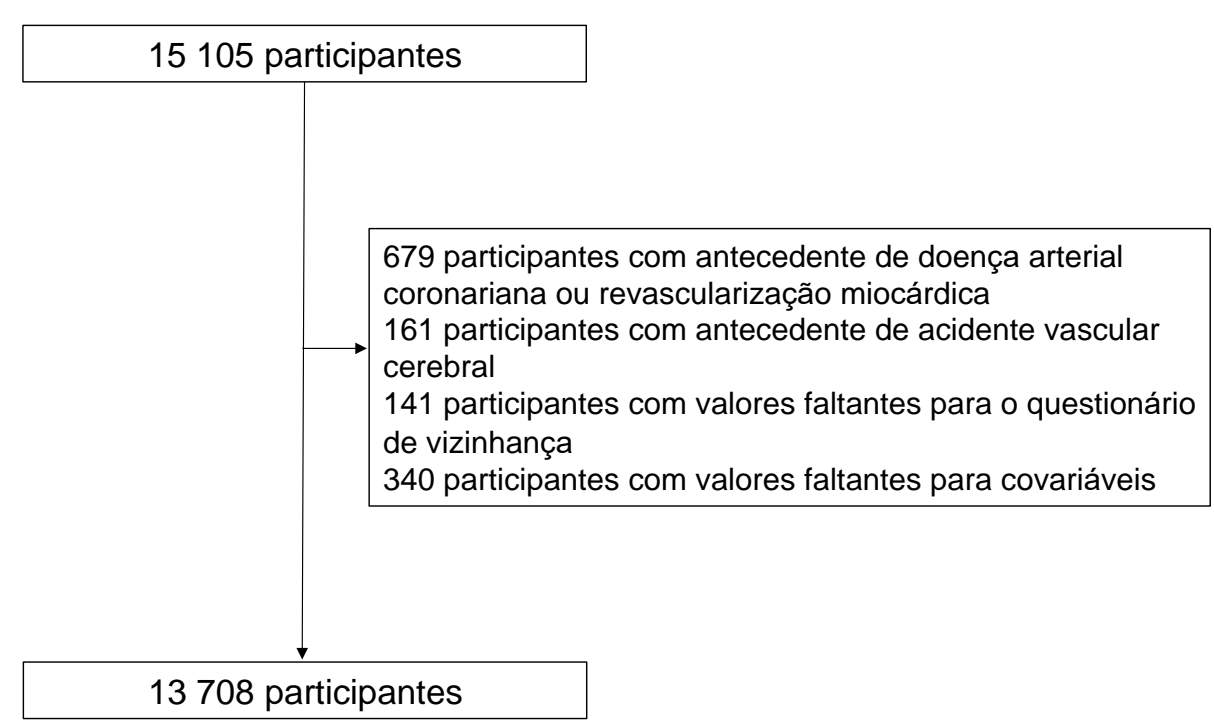

Figura 6 - Fluxograma de participantes para o modelo de imputação múltipla para valores ausentes ("missings") da espessura íntima-média de carótidas

Quando imputamos os dados de EIMC utilizando o método de imputação múltipla, melhor percepção de ambiente para atividade física ( $\beta=$ $0,46$, IC $95 \%=-0,85 ;-0,06, p=0,02)$ e baixa percepção de violência $(\beta=-1,52$, IC $95 \%=-2,50 ;-0,55, p=0,002$ ) estiveram associadas com menor EIMC, na 
análise ajustada por idade, sexo e anos de residência na vizinhança (Tabela 6). Por outro lado, baixa percepção de vitimização pessoal esteve associada com maior EIMC $(\beta=7,78$, IC $95 \%=2,09 ; 13,46, p=0,007)$. Quando ajustamos este modelo para cor, variáveis socioeconômicas e de risco cardiovascular, nenhuma associação foi encontrada entre os domínios e a EIMC. 
Tabela 6 - Coeficientes e intervalos de confiança (IC) de 95\% para EIMC $(\mu \mathrm{m})$ de acordo com percepção de características de vizinhança $(n=9.923)$

\begin{tabular}{llccc}
\hline $\begin{array}{l}\text { DOMÍNIO DE } \\
\text { CARACTERÍSTICAS } \\
\text { DE VIZINHANÇA }\end{array}$ & Crua & Modelo 1 & Modelo 2 & Modelo 3 \\
\hline & $\beta\left(95 \% \mathrm{Cl}^{+}\right)$ & $\beta\left(95 \% \mathrm{Cl}^{+}\right)$ & $\beta\left(95 \% \mathrm{Cl}^{+}\right)$ & $\beta\left(95 \% \mathrm{Cl}^{+}\right)$ \\
\cline { 2 - 5 } $\begin{array}{l}\text { Coesão social } \\
\begin{array}{l}\text { Ambiente para } \\
\text { atividade física }\end{array}\end{array}$ & $0,94(0,08 ; 1.80)$ & $-0,55(-1,34 ; 0,24)$ & $-0,16(-0,95 ; 0,64)$ & $0,01(-0,76 ; 0,79)$ \\
$\begin{array}{l}\text { Disponibilidade de } \\
\text { alimento saudável }\end{array}$ & $0,68(1,53 ; 3,83)$ & $0,22(-0,82 ; 1,26)$ & $0,50(-0,55 ; 1,56)$ & $0,58(-0,46 ; 1,62)$ \\
$\begin{array}{l}\text { Segurança } \\
\text { Violência percebida }\end{array}$ & $1,84(0,83 ; 2,85)$ & $-0,03(-0,96 ; 0,90)$ & $-0,11(-1,04 ; 0,82)$ & $-0,19(1,10 ; 0,72)$ \\
Vitimização pessoal & $8,01(1,72 ; 14,30)$ & $7,78(2,09 ; 13,46)$ & $4,37(-1,32 ; 5,73)$ & $3,08(-2,48 ; 8,63)$
\end{tabular}

NOTA: Valores ausentes ("missings") para EIMC foram substituídos por imputação múltipla

Modelo 1: Regressão linear ajustada por idade, sexo e anos de residência

Modelo 2: Regressão linear ajustada por idade, sexo, anos de residência, cor, renda e educação

Modelo 3: Regressão linear ajustada por idade, sexo, anos de residência, cor, renda, educação,

hipertensão, diabetes. Dislipidemia, tabagismo, abuso de álcool, atividade física e IMC 
Tabela 7 - Coeficientes e intervalos de confiança (IC) de 95\% para EIMC $(\mu \mathrm{m})$ de acordo com percepção de características de vizinhança, estratificado por anos de residência $(n=9.923)$

\begin{tabular}{lccl}
\hline $\begin{array}{l}\text { DOMÍNIO DE } \\
\text { CARACTERÍSTICAS DE } \\
\text { VIZINHANÇA }\end{array}$ & Modelo 1 & Modelo 2 & Modelo 3 \\
\hline & & & \\
\cline { 2 - 4 } $\begin{array}{l}\text { Coesão social } \\
5 \text { anos ou menos }\end{array}$ & $-0,59(-2,19 ; 1,21)$ & $-0,14(-1,82 ; 1,54)$ & $0,07(-0,16 ; 1,72)$ \\
Mais de 5 anos & $-0,55(-1,46 ; 0,37)$ & $-0,15(-1,06 ; 0,76)$ & $0,00(-0,90 ; 0,88)$ \\
$\begin{array}{l}\text { Ambiente para atividade } \\
\text { física }\end{array}$ & & & \\
5 anos ou menos & $0,17(-0,50 ; 0,83)$ & $0,41(-0,27 ; 1,09)$ & $0,53(-0,14 ; 1,20)$ \\
$\begin{array}{l}\text { Mais de } 5 \text { anos } \\
\text { Disponibilidade de alimento } \\
\text { saudável }\end{array}$ & $-0,74(-1,22 ;-0,25)$ & $-0,43(-0,92 ; 0,05)$ & $-0,31(-0,79 ; 0,17)$ \\
5 anos ou menos & & & \\
Mais de 5 anos & $-0,35(-2,17 ; 1,46)$ & $-0,16(-1,99 ; 1,66)$ & $0,05(-1,76 ; 1,86)$ \\
Segurança & $0,41(-0,83 ; 1,66)$ & $0,77(-0,49 ; 2,03)$ & $0,78(-0,46 ; 2,01)$ \\
5 anos ou menos & & & \\
Mais de 5 anos & $-0,38(-1,95 ; 1,17)$ & $-0,41(-1,97 ; 0,12)$ & $-0,53(-2,08 ; 1,02)$ \\
\hline
\end{tabular}




\section{Violência percebida}

5 anos ou menos

$-2,17(-4,19 ; 0,15)$

$-1,54(-3,57 ; 0,49)$

$-1,03(-3,02 ; 0,96)$

Mais de 5 anos

$-1,47(-2,63 ;-0,31)$

$-0,62(-1,78 ; 0,54)$

$-0,37(-1,51 ; 0,76)$

\section{Vitimização pessoal}

5 anos ou menos

$$
0,07(-13,55 ; 13,69) \quad-0,48(-14,09 ; 13,12) \quad-1,98(-15,37 ; 11,40)
$$

Mais de 5 anos

$8,10(1,48 ; 14,73)$

$4,36(-2,34 ; 11,06)$

$3,38(-3,17 ; 9,94)$

NOTA: Valores ausentes ("missings") para EIMC foram substituídos por imputação múltipla

Modelo 1: Regressão linear ajustada por idade e sexo

Modelo 2: Regressão linear ajustada por idade, sexo, cor, renda e educação

Modelo 3: Regressão linear ajustada por idade, sexo, cor, renda, educação, hipertensão, diabetes,

dislipidemia, tabagismo, abuso de álcool, atividade física e IMC 
5. Discussão 
Em uma grande amostra brasileira de adultos livres de DCV clínica, a EIMC esteve negativamente associada à boa percepção de ambiente para atividade física e à baixa violência percebida em nível de vizinhança e positivamente associada com percepção de baixa vitimização pessoal na análise ajustada por variáveis demográficas. Essas associações não se mantiveram significantes após o ajuste para fatores socioeconômicos individuais e de risco cardiovascular. A análise estratificada por anos de residência confirmou esses resultados entre aqueles participantes que viviam na vizinhança examinada havia 5 anos ou mais. O modelo de imputação múltipla para dados faltantes de EIMC também reforçou os resultados. Apesar de estudos anteriores sobre associações de características de vizinhança e aterosclerose subclínica terem verificado diferenças entre homens e mulheres $(25,30,31)$, nós encontramos, dentre todos os domínios avaliados, apenas uma interação limítrofe entre sexo e percepção de coesão social e segurança. $\mathrm{Na}$ análise estratificada, apenas homens mostraram uma associação protetora entre EIMC e melhor percepção de segurança.

\subsection{Comparação com resultados de estudos anteriores}

O presente trabalho traz resultados inéditos e sua comparação com publicações anteriores deve levar em consideração que este é o primeiro estudo a verificar associação entre a percepção de domínios específicos de 
vizinhança e aterosclerose subclínica medida por EIMC. Os poucos estudos que investigaram a associação entre aterosclerose subclínica e características de vizinhança utilizaram status socioeconômico, posição socioeconômica e pobreza como indicadores de vizinhança e/ou escore de cálcio de artérias coronarianas como medida de aterosclerose subclínica(20,22,23,25,26,30).

O fato de a associação entre a percepção de características de vizinhança não ter ocorrido nos modelos ajustados por fatores de risco socioeconômicos individuais e cardiovasculares está de acordo com publicações anteriores $(22,25,30)$ e pode indicar que tais fatores têm um papel mediador na associação. Em um estudo conduzido entre homens hipertensos livres de DCV, a média da EIMC era maior entre aqueles residentes em áreas mais desfavorecidas do que aqueles que viviam em vizinhanças mais ricas. No entanto, quando o status socioeconômico da comunidade foi adicionado aos modelos após inclusão de status socioeconômico individual como preditor, a associação não se manteve. O presente estudo também está de acordo com análises prévias do estudo MESA, o qual encontrou associação entre pobreza em nível de vizinhança e EIMC entre mulheres, mas a associação foi atenuada após ajuste para fatores de risco cardiovasculares (30). Outros autores investigaram pobreza na vizinhança na mesma coorte, medindo trajetórias de vizinhança ao longo do tempo e sua associação com EIMC (25). Em modelos ajustados por idade, alta pobreza acumulada esteve associada com piores desfechos cardiovasculares, inclusive EIMC principalmente entre mulheres. Tal 
associação passou a ser limítrofe após ajustes para raça/etnia e posição socioeconômica na vida adulta.

A seguir, discutem-se particularidades dos resultados encontrados neste estudo para cada domínio de percepção de vizinhança avaliado.

\subsection{Coesão social}

A hipótese de que a percepção de um ambiente de vizinhança com maior coesão social estaria associada a menor EIMC foi refutada nas análises aqui apresentadas. Tal hipótese partiu da ideia de que uma vizinhança coesa socialmente oferta ao morador um ambiente menos estressor (Figura 3). De fato, trabalhos anteriores detectaram associação entre coesão social e hipertensão (45), entre coesão social e transtorno mental comum (32), entre percepção de coesão social e infarto do miocárdio (46) e entre percepção de coesão social e acidente vascular cerebral (47). Um estudo recente encontrou relação entre percepção de coesão social e uso de serviços preventivos de saúde (48), como, por exemplo, busca por realização de teste de níveis séricos de colesterol.

De forma alinhada com nossa hipótese, o estudo Coronary Artery Disease Risk Development in Young Adults (CARDIA), encontrou relação entre coesão social e ECAC em mulheres jovens, utilizando as mesmas perguntas por nós utilizadas (31). Porém, a faixa etária da população estudada por CARDIA era 
mais jovem que a nossa (adultos entre 33 e 50 anos) e a medida de ECAC desse estudo foi realizada cinco anos após a exposição.

A comparação dos resultados de CARDIA com os resultados da amostra do ELSA aqui apresentados sugere que coesão social pode agir como fator protetor na fase mais inicial da formação de placas, ou ainda, que as populações mais jovens dependam mais das relações de suas vizinhanças do que populações de trabalhadores mais velhos como os encontrados em nossa amostra (Tabela 1), que estabelecem vínculos mais intensos com colegas de trabalho e lá formam redes de apoio, tornando as relações da vizinhança menos importantes do ponto de vista da geração e diminuição do estresse psicossocial.

\subsection{Ambiente para atividade física}

As análises desta tese confirmaram a hipótese de que a percepção de viver em uma vizinhança com ambiente mais propício à atividade física estivesse associada a menor EIMC. Os mecanismos pelos quais essa associação se dá podem ter relação com maior facilidade à pratica de exercícios em momentos de lazer e menor sedentarismo por maior atividade física em atividades rotineiras, o que, por sua vez, pode interferir com fatores de risco biológicos tais como pressão arterial, IMC, diabetes mellitus, dislipidemias, maior nível de estresse psicossocial, entre outros (Figura 3). 
De fato, viver em um ambiente mais propício à atividade física já havia sido associada à prática de exercícios, à perda de peso medida por IMC e à apneia obstrutiva do sono em estudos anteriores $(37,49-51)$.

\subsection{Disponibilidade de alimento saudável}

O presente estudo não confirmou a hipótese de que a percepção sobre boa disponibilidade de alimento saudável na vizinhança estivesse relacionada a menores EIMC nesta população.

Publicações prévias de países ricos verificaram relação entre a percepção da maior disponibilidade de alimento saudável na vizinhança e qualidade dietética $(34,52,53)$ e diabetes tipo $2(54,55)$, o que vai ao encontro da hipótese dentro do modelo esquemático conceitual proposto das relações entre as características de vizinhança e DCV (Figura 3).

Uma possível explicação para a ausência da relação no presente trabalho é que haja uma diferença entre o comportamento de compra do brasileiro em relação aos consumidores de países ricos. A percepção da disponibilidade de alimento saudável nas regiões em que os participantes moram pode não ser tão importantes na determinação do que consomem por duas razões.

Os brasileiros de grandes metrópoles, comumente, se dispõem a realizar compras de alimentos em grandes redes de supermercados distantes de suas 
residências, onde o preço é mais baixo. Esse hábito tem relação com o fato de que os salários, aqui, são pagos mensalmente, o que favorece o hábito do estoque de comidas. Além disso, muitos dos participantes desta amostra viveram o período de hiperinflação dos anos 1980 e 1900, quando era preciso fazer grandes compras para defender-se das altas dos preços. Os norteamericanos - população na qual foi encontrada associação entre a percepção da disponibilidade e o consumo de alimento saudável(34), por outro lado, podem ter hábitos de consumo diferentes, utilizando mais os recursos da própria região de residência.

Outra possível explicação para não ter sido encontrada associação entre a percepção da disponibilidade de alimento saudável e EIMC é que, por serem trabalhadores de universidades localizadas em grandes metrópoles, os participantes não costumem realizar muitas refeições nem as principais compras de alimentos próximo às suas casas. A dinâmica de ocupação e deslocamento dentro das metrópoles brasileiras, muitas vezes, faz com que o trabalhador passe muito pouco tempo em sua própria vizinhança e termine realizando suas principais atividades no entorno do local de trabalho ou nos próprios refeitórios da universidade.

\subsection{Segurança}


Não foi confirmada a associação entre a percepção de segurança e a EIMC. Este resultado negativo contrasta com o resultado encontrado no domínio "violência percebida", que esteve associado a menores EIMC.

Analisando e comparando às perguntas que compõem cada um desses dois domínios (Anexo, Quadro 1), percebemos que as perguntas do domínio "segurança" são mais abertas e interpretativas do que as do domínio "violência percebida". Isso pode indicar que em países com altos índices de violência como o Brasil, ocorre uma naturalização da violência: ao serem questionados de forma aberta e interpretativa sobre a opinião sobre as suas vizinhanças, os participantes relatam uma percepção melhor do que quando são feitas perguntas mais objetivas. Os acontecimentos violentos de fatos objetivos são os que determinam o nível de atividade física e estresse dos indivíduos.

\subsection{Violência percebida}

Foi confirmada a hipótese de que perceber a vizinhança em que se mora como menos violenta estivesse associado a menor EIMC. O que significa que, perceber sua vizinhança como um local com menos episódios de brigas, discussões violentas, assaltos, estupros ou roubos pode oferecer proteção nas fases iniciais do processo de aterosclerose.

Estudos anteriores do MESA descreveram associações entre outros domínios relacionados à violência e menor IMC (35), hipertensão (33) e 
maiores níveis de caminhabilidade (38) em Chicago (Estados Unidos), reforçando o modelo conceitual das relações entre os aspectos de vizinhança relacionados ao crime, violência e segurança e DCV (Figura 3).

\subsection{Vitimização pessoal}

O presente estudo encontrou resultados contra-intuitivos a respeito deste domínio: a melhor percepção de vitimização pessoal esteve positivamente associada à EIMC.

A hipótese de que a percepção sobre viver em vizinhanças em que as pessoas sofram menos episódios de violência estivessem associadas a menores EIMC parte do raciocínio de que viver neste ambiente cause menos estresse psicossocial (Figura 3) e que impacte no nível de atividade física das pessoas, favorecendo a caminhabilidade, pelo melhor uso do espaço público. De fato, em trabalhos anteriores, vizinhanças caracterizadas como mais violentas foram associadas com menores níveis séricos de cortisol ao acordar, menor declínio matinal e declive mais plano entre a hora de acordar e de dormir $(56,57)$. Uma linha de pesquisa intrigante tem postulado que estresse crônico poderia afetar a saúde cardiovascular por impacto no encurtamento de telômeros (58) e estudos do MESA encontraram menores telômeros em participantes que viviam em vizinhanças caracterizadas como piores esteticamente, menos seguras e com pior coesão social. 
Diante disso, é possível que este resultado inesperado seja resultado de erro tipo 1 (falso positivo), decorrente das múltiplas comparações feitas no estudo. Outra possibilidade é que haja uma variável de confusão (ser casado/a) interferindo e causando a associação. Para compreender isso, é preciso observar que este domínio é, na verdade, composto de uma única pergunta, transcrita a seguir:

"Alguém já praticou violência (assalto, briga, violência sexual ou sequestro) contra o(a) senhor(a) ou contra algum morador de sua residência, nessa vizinhança, durante o tempo em que o(a) senhor(a) mora nesse local?" (Anexo, Quadro1)

Ao analisar esta pergunta, compreende-se que o participante que respondeu "sim" pode ter se referido a si mesmo ou a algum morador de sua residência. Logo, aqueles que moram com mais pessoas tiveram maior chance de responder "sim" a essa pergunta. Dadas as características da amostra (Tabela 1) e a maior frequência de episódios de violência em jovens, é provável que aqueles que responderam sim sejam mães ou pais de família com filhos jovens e que têm, portanto, mais chance de serem casados.

\subsection{Forças do estudo}


Pelo que pode-se apurar até a publicação deste trabalho, este é o maior estudo que investigou associações entre fatores de risco contextuais em nível de vizinhança e aterosclerose subclínica. O grande tamanho da amostra permitiu que fossem examinadas interações com sexo e realizadas análises estratificadas por tempo de residência.

Este é também o trabalho que investigou associação entre DCV e maior número de domínios ao mesmo tempo. Foram analisados seis domínios diferentes de características de vizinhança. A maioria dos estudos avalia apenas um a três domínios diferentes(32,33,35,37).

A utilização de EIMC para acessar aterosclerose subclínica também consiste em um ponto forte do presente trabalho, uma vez que permitiu que fossem identificadas interferências da percepção de vizinhança em fases precoces da formação de placa, quando há mais oportunidades para intervenções preventivas no nível populacional $(22,25,28,30,31,59)$.

Ademais, este é também o primeiro estudo a avaliar fatores de risco contextuais e aterosclerose subclínica em um país pobre. Informação proveniente de países em desenvolvimento é escassa e muito importante pela grande carga de doença, grande disparidade e prevalência de risco nesses países $(1,60)$.

\subsection{Limitações do estudo}


Algumas limitações relacionadas à amostra e às análises precisam ser consideradas na interpretação dos resultados. A primeira é que a amostra investigada corresponde a indivíduos de uma coorte de trabalhadores de universidades públicas, que não é representativa da população brasileira no que diz respeito às condições socioeconômicas, o que pode limitar a capacidade de generalização dos resultados. No entanto, é importante destacar que a amostra do ELSA-Brasil tem frequência de fatores de risco cardiovasculares e estilo de vida semelhantes à da população Brasileira (61).

Outra limitação diz respeito à incerteza sobre se, de fato, o participante utilizava os recursos de sua vizinhança para realizar as atividades correspondentes àquele recurso avaliado em determinado domínio. Como descrito na seção de Métodos, antes de começar a responder às perguntas do questionário, o participante foi convidado a "pensar sua vizinhança como a área geral do entorno da residência onde o/a senhor/senhora costuma realizar atividades de rotina como, por exemplo, fazer compras, ir ao parque ou visitar vizinhos". No entanto considerando a dinâmica de ocupação e deslocamento das grandes metrópoles, é comum que as pessoas utilizem o entorno de seu local de trabalho para fazer compras, atividades físicas e de lazer, passando a maior parte do tempo nessas regiões do que na vizinhança em que vivem. Nós não obtivemos informações dos locais de trabalho dos participantes, que 
podem ser igualmente ou mais importantes fatores contextuais de um ou mais domínios avaliados.

Ademais, as informações de endereço e de aterosclerose subclínica foram obtidas uma única vez e simultaneamente, visto que este é um estudo transversal. Esse fato pode resultar em medidas imprecisas da influência do ambiente, uma vez que o desenvolvimento de aterosclerose é lento e cumulativo ao longo da vida(3,4).

Por fim, as medidas de EIMC estavam ausentes em $26 \%$ dos participantes da amostra original. De fato, houve muitas perdas durante o processo de aquisição de imagens de EIMC. No entanto, a perda não foi direcionada a qualquer grupo (perdas ao acaso). Ela se deu principalmente pela indisponibilidade do aparelho de ultrassonografia no mesmo dia de outros procedimentos da avaliação da linha de base do estudo em alguns centros em alguns dos dias, obrigando o retorno dos participantes em outro dia para realizá-lo, o que não ocorreu sempre. De qualquer maneira, para minimizar o potencial efeito dessas perdas, foram incluídas análises utilizando imputação múltipla para os dados faltantes de EIMC, encontrando resultados similares. 
7. Conclusões 
- A percepção sobre viver em vizinhança com melhor ambiente para atividade física esteve associada a menor EIMC. Fatores de risco socioeconômicos e de risco cardiovascular podem ter papel mediador.

- A percepção sobre viver em vizinhança com menor violência esteve associada a menor EIMC. Fatores de risco socioeconômicos individuais e de risco cardiovascular podem ter papel mediador.

- A percepção de viver em uma vizinhança com baixa vitimização pessoal esteve associada a maior EIMC.

- Não houve associação entre a percepção sobre coesão social, disponibilidade de alimento saudável ou vitimização pessoal e EIMC.

Os resultados desta tese foram publicados no periódico Global Heart em dezembro de 2019(62) (Anexos). 
8. Referências 
1. WHO. Noncommunicable Diseases Country Profiles 2018. World Health Organization. 2018. 223 p.

2. BRASIL. Ministério da Saúde. Secretariade Vigilância em Saúde. Coordenação-Geral de Informações e Análises Epidemiológicas. DATASUS [Internet]. [citado 2 de dezembro de 2019]. Available at: http:/ttabnet.datasus.gov.br/cgi/tabcgi.exe?sim/cnv/obt10br.def

3. Kumar V. Patologia - Bases patológicas das doenças. $9^{\circ}$ ed. Elsevier; 2016. $1440 \mathrm{p}$.

4. Polak JF, Pencina MJ, Pencina KM, O'Donnell CJ, Wolf PA, D'Agostino RBS. Carotid-wall intima-media thickness and cardiovascular events. N Engl J Med. julho de 2011;365(3):213-21.

5. Nambi V, Chambless L, Folsom AR, He M, Hu Y, Mosley T, et al. Carotid Intima-Media Thickness and Presence or Absence of Plaque Improves Prediction of Coronary Heart Disease Risk. The ARIC (Atherosclerosis Risk In Communities) Study. J Am Coll Cardiol [Internet]. 2010;55(15):1600-7.

Available

at:

http://dx.doi.org/10.1016/j.jacc.2009.11.075

6. Lorenz MW, Polak JF, Kavousi M, Mathiesen EB, Völzke H, Tuomainen TP, et al. Carotid intima-media thickness progression to predict cardiovascular events in the general population (the PROG-IMT collaborative project): A meta-analysis of individual participant data. Lancet [Internet]. 2012;379(9831):2053-62. Available at: http://dx.doi.org/10.1016/S0140-6736(12)60441-3

7. van den Oord SCH, Sijbrands EJG, ten Kate GL, van Klaveren D, van Domburg RT, van der Steen AFW, et al. Carotid intima-media thickness for cardiovascular risk assessment: systematic review and meta-analysis. Atherosclerosis. maio de 2013;228(1):1-11.

8. Polak JF, Szklo M, Kronmal RA, Burke GL, Shea S, Zavodni AEH, et al. 
The value of carotid artery plaque and intima-media thickness for incident cardiovascular disease: the multi-ethnic study of atherosclerosis. J Am Heart Assoc. 2013;2(2):1-10.

9. Vliegenthart R, Oudkerk M, Hofman A, Oei HHS, Van Dijck W, Van Rooij FJA, et al. Coronary calcification improves cardiovascular risk prediction in the elderly. Circulation. 2005;112(4):572-7.

10. Bensenor IM, Goulart AC, Santos IS, Bittencourt MS, Pereira AC, Santos $\mathrm{RD}$, et al. Association between a healthy cardiovascular risk factor profile and coronary artery calcium score: Results from the Brazilian Longitudinal Study of Adult Health (ELSA-Brasil). Am Heart J [Internet]. 2016;174:519. Available at: http://dx.doi.org/10.1016/j.ahj.2015.12.018

11. Detrano R, Guerci AD, Carr JJ, Bild DE, Burke G, Folsom AR, et al. Coronary calcium as a predictor of coronary events in four racial or ethnic groups. N Engl J Med. março de 2008;358(13):1336-45.

12. Raggi P, Callister TQ, Cooil B, He ZX, Lippolis NJ, Russo DJ, et al. Identification of patients at increased risk of first unheralded acute myocardial infarction by electron-beam computed tomography. Circulation. 2000;101(8):850-5.

13. Newstein D, Arad Y, Guerci AD, Spadaro LA, Goodman K. Prediction of coronary events with electron beam computed tomography. J Am Coll Cardiol [Internet]. 2002;36(4):1253-60. Available at: http://dx.doi.org/10.1016/S0735-1097(00)00872-X

14. Kondos GT, Hoff JA, Sevrukov A, Daviglus ML, Garside DB, Devries SS, et al. Electron-beam tomography coronary artery calcium and cardiac events: a 37-month follow-up of 5635 initially asymptomatic low- to intermediate-risk adults. Circulation. maio de 2003;107(20):2571-6.

15. Greenland P, LaBree L, Azen SP, Doherty TM, Detrano RC. Coronary artery calcium score combined with Framingham score for risk prediction 
in asymptomatic individuals. JAMA. janeiro de 2004;291(2):210-5.

16. Gepner AD, Young R, Delaney JA, Tattersall MC, Blaha MJ, Post WS, et al. Comparison of coronary artery calcium presence, carotid plaque presence, and carotid intima-media thickness for cardiovascular disease prediction in the multi-ethnic study of atherosclerosis. Circ Cardiovasc Imaging. 2014;8(1).

17. Rose G. Sick individuals and sick populations. Bull World Health Organ. $2001 ; 79(10): 990-6$.

18. Diez-Roux A V. Investigating Neighborhood and Area Effects on Health. Am J Public Health. 2001;91(11):1783-9.

19. Diez Roux A V, Mujahid MS, Hirsch JA, Moore K, Moore L V. The Impact of Neighborhoods on CV Risk. Glob Heart. setembro de 2016;11(3):35363.

20. Carson AP, Rose KM, Catellier DJ, Kaufman JS, Wyatt SB, Diez-Roux A $\mathrm{V}$, et al. Cumulative socioeconomic status across the life course and subclinical atherosclerosis. Ann Epidemiol. abril de 2007;17(4):296-303.

21. Nordstrom CK, Diez Roux A V, Jackson SA, Gardin JM. The association of personal and neighborhood socioeconomic indicators with subclinical cardiovascular disease in an elderly cohort. The cardiovascular health study. Soc Sci Med. novembro de 2004;59(10):2139-47.

22. Petersen KL, Bleil ME, McCaffery J, Mackey $\mathrm{RH}$, Sutton-Tyrrell $\mathrm{K}$, Muldoon MF, et al. Community Socioeconomic status is associated with artery atherosclerosis in untreated, hypertensive men. Am J Hypertens. junho de 2006;19(6):560-6.

23. Rosvall M, Engström G, Hedblad B, Janzon L, Berglund G. Area social characteristics and carotid atherosclerosis. Eur $J$ Public Health. 2007;17(4):333-9. 
24. Chaix B. Geographic Life Environments and Coronary Heart Disease: A Literature Review, Theoretical Contributions, Methodological Updates, and a Research Agenda. Annu Rev Public Health. 2009;30(1):81-105.

25. Murray ET, Diez Roux A V, Carnethon M, Lutsey PL, Ni H, O'Meara ES. Trajectories of neighborhood poverty and associations with subclinical atherosclerosis and associated risk factors: the multi-ethnic study of atherosclerosis. Am J Epidemiol. maio de 2010;171(10):1099-108.

26. Diez-Roux A V. Change in Neighborhood Characteristics and Change in Coronary Artery Calcium. Circulation. 2016;134(7):504-13.

27. Flores-Torres MH, Lynch R, Lopez-Ridaura R, Yunes E, Monge A, OrtizPanozo E, et al. Exposure to Violence and Carotid Artery Intima-Media Thickness in Mexican Women. J Am Heart Assoc. agosto de 2017;6(8).

28. Wing JJ, August E, Adar SD, Dannenberg AL, Hajat A, Sanchez BN, et al. Change in Neighborhood Characteristics and Change in Coronary Artery Calcium: A Longitudinal Investigation in the MESA (Multi-Ethnic Study of Atherosclerosis) Cohort. Circulation. agosto de 2016;134(7):504-13.

29. Grimaud O, Lapostolle A, Berr C, Helmer C, Dufouil C, Kihal W, et al. Gender differences in the association between socioeconomic status and subclinical atherosclerosis. PLoS One. 2013;8(11):e80195.

30. Lemelin ET, Diez Roux A V, Franklin TG, Carnethon M, Lutsey PL, Ni H, et al. Life-course socioeconomic positions and subclinical atherosclerosis in the multi-ethnic study of atherosclerosis. Soc Sci Med. fevereiro de $2009 ; 68(3): 444-51$.

31. Kim D, Diez Roux A V, Kiefe Cl, Kawachi I, Liu K. Do neighborhood socioeconomic deprivation and low social cohesion predict coronary calcification?: the CARDIA study. Am J Epidemiol. agosto de 2010;172(3):288-98. 
32. Secretti T, Nunes MAA, Schmidt MI, Stein MC, Santos SM. Characteristics of neighborhood environment (social cohesion and safety) and common mental disorders in ELSA-Brasil study: a multilevel analysis. Cad Saude Publica. 2019;35(1):e00197017.

33. Mayne SL, Moore KA, Powell-Wiley TM, Evenson KR, Block R, Kershaw KN. Longitudinal Associations of Neighborhood Crime and Perceived Safety With Blood Pressure: The Multi-Ethnic Study of Atherosclerosis (MESA). Am J Hypertens. agosto de 2018;31(9):1024-32.

34. Kern DM, Auchincloss AH, Stehr MF, Roux AVD, Moore L V, Kanter GP, et al. Neighborhood Prices of Healthier and Unhealthier Foods and Associations with Diet Quality: Evidence from the Multi-Ethnic Study of Atherosclerosis. Int $\mathrm{J}$ Environ Res Public Health. novembro de $2017 ; 14(11)$.

35. Powell-Wiley TM, Moore K, Allen N, Block R, Evenson KR, Mujahid M, et al. Associations of Neighborhood Crime and Safety and With Changes in Body Mass Index and Waist Circumference: The Multi-Ethnic Study of Atherosclerosis. Am J Epidemiol. agosto de 2017;186(3):280-8.

36. Kerr Z, Evenson KR, Moore K, Block R, Diez Roux A V. Changes in walking associated with perceived neighborhood safety and policerecorded crime: The multi-ethnic study of atherosclerosis. Prev Med (Baltim). abril de 2015;73:88-93.

37. Hirsch JA, Diez Roux A V, Moore KA, Evenson KR, Rodriguez DA. Change in walking and body mass index following residential relocation: the multi-ethnic study of atherosclerosis. Am J Public Health. março de 2014;104(3):e49-56.

38. Evenson KR, Block R, Diez Roux A V, McGinn AP, Wen F, Rodriguez DA. Associations of adult physical activity with perceived safety and police-recorded crime: the Multi-ethnic Study of Atherosclerosis. Int $\mathrm{J}$ 
Behav Nutr Phys Act. dezembro de 2012;9:146.

39. Echeverria SE, Diez-Roux A V., Link BG. Reliability of self-reported neighborhood characteristics. J Urban Heal. 2004;81(4):682-701.

40. Mill JG, Pinto K, Griep RH, Goulart A, Foppa M, Lotufo P, et al. Medical assessments and measurements in ELSA-Brasil. Rev Saude Publica. 2013;47(2):54-62.

41. AQUINO ML_ELSA-Brasil Objectives and Design_AmJElidemiol_2012.

42. Santos SM, Griep RH, Cardoso LO, Alves MG de M, Fonseca M de JM da, Giatti L, et al. Adaptacao transcultural e confiabilidade de medidas de caracteristicas autorreferidas de vizinhanca no ELSA-Brasil. Rev Saude Publica. 2013;47(suppl 2):122-30.

43. Santos IS, Bittencourt MS, Oliveira IRS, Souza AG, Meireles DP, Rundek $\mathrm{T}$, et al. Carotid intima-media thickness value distributions in The Brazilian Longitudinal Study of Adult Health (ELSA-Brasil). Atherosclerosis. 2014;237(1):227-35.

44. StataCorp. Multiple-imputation reference manual, release 13. Stata Press, StataCorpLP, College stations, Texas. 2013.

45. Lagisetty PA, Wen M, Choi H, Heisler M, Kanaya AM, Kandula NR. Neighborhood Social Cohesion and Prevalence of Hypertension and Diabetes in a South Asian Population. J Immigr Minor Heal. dezembro de 2016;18(6):1309-16.

46. Kim ES, Hawes AM, Smith J. Perceived neighbourhood social cohesion and myocardial infarction. J Epidemiol Community Health. novembro de 2014;68(11):1020-6.

47. Kim ES, Park N, Peterson C. Perceived neighborhood social cohesion and stroke. Soc Sci Med. novembro de 2013;97:49-55.

48. Kim ES, Kawachi I. Perceived Neighborhood Social Cohesion and 
Preventive Healthcare Use. Am J Prev Med. agosto de 2017;53(2):e3540.

49. Barrientos-Gutierrez T, Moore KAB, Auchincloss AH, Mujahid MS, August C, Sanchez BN, et al. Neighborhood Physical Environment and Changes in Body Mass Index: Results From the Multi-Ethnic Study of Atherosclerosis. Am J Epidemiol. dezembro de 2017;186(11):1237-45.

50. Billings ME, Johnson DA, Simonelli G, Moore K, Patel SR, Diez Roux A $V$, et al. Neighborhood Walking Environment and Activity Level Are Associated With OSA: The Multi-Ethnic Study of Atherosclerosis. Chest. novembro de 2016;150(5):1042-9.

51. Chor D, Cardoso LO, Nobre AA, Griep RH, Fonseca M de JM, Giatti L, et al. Association between perceived neighbourhood characteristics, physical activity and diet quality: results of the Brazilian Longitudinal Study of Adult Health (ELSA-Brasil). BMC Public Health. 2016;16(1):111.

52. Moore L V, Diez Roux A V, Nettleton JA, Jacobs DR, Franco M. Fast-food consumption, diet quality, and neighborhood exposure to fast food: the multi-ethnic study of atherosclerosis. Am $\mathrm{J}$ Epidemiol. julho de 2009;170(1):29-36.

53. Morland K, Wing S, Diez Roux A. The contextual effect of the local food environment on residents' diets: the atherosclerosis risk in communities study. Am J Public Health. novembro de 2002;92(11):1761-7.

54. Kern DM, Auchincloss AH, Stehr MF, Diez Roux A V, Moore KA, Kanter GP, et al. Neighborhood price of healthier food relative to unhealthy food and its association with type 2 diabetes and insulin resistance: The multiethnic study of atherosclerosis. Prev Med (Baltim). janeiro de 2018;106:122-9.

55. Auchincloss AH, Diez Roux A V, Mujahid MS, Shen M, Bertoni AG, 
Carnethon MR. Neighborhood resources for physical activity and healthy foods and incidence of type 2 diabetes mellitus: the Multi-Ethnic study of Atherosclerosis. Arch Intern Med. outubro de 2009;169(18):1698-704.

56. Do DP, Diez Roux A V, Hajat A, Auchincloss AH, Merkin SS, Ranjit N, et al. Circadian rhythm of cortisol and neighborhood characteristics in a population-based sample: the Multi-Ethnic Study of Atherosclerosis. Health Place. março de 2011;17(2):625-32.

57. Hajat A, Moore K, Do DP, Stein Merkin S, Punjabi NM, Sanchez BN, et al. Examining the cross-sectional and longitudinal association between diurnal cortisol and neighborhood characteristics: Evidence from the multi-ethnic study of atherosclerosis. Health Place. julho de 2015;34:199206.

58. Nilsson PM, Tufvesson H, Leosdottir M, Melander O. Telomeres and cardiovascular disease risk: an update 2013. Transl Res. dezembro de 2013;162(6):371-80.

59. Bentzon JF, Otsuka F, Virmani R, Falk E. Mechanisms of plaque formation and rupture. Circ Res. junho de 2014;114(12):1852-66.

60. Schmidt MI, Duncan BB, Azevedo e Silva G, Menezes AM, Monteiro CA, Barreto SM, et al. Chronic non-communicable diseases in Brazil: burden and current challenges. Lancet (London, England). junho de 2011;377(9781):1949-61.

61. Schmidt MI, Duncan BB, Mill JG, Lotufo PA, Chor D, Barreto SM, et al. Cohort Profile: Longitudinal Study of Adult Health (ELSA-Brasil). Int J Epidemiol. fevereiro de 2015;44(1):68-75.

62. Willets C, Santos IS, Lotufo PA, Bensenor IM, Suemoto CK. Association Between Perceived Neighborhood Characteristics and Carotid Artery Intima-Media Thickness: Cross-Sectional Results From the ELSA-Brasil Study. Glob Heart. dezembro de 2019;14(4):379-85. 


\section{Anexos}

Quadro 1 - Questionário de percepção de características de vizinhança do ELSA-Brasil.

\begin{tabular}{|c|c|}
\hline DOMÍNIO & Perguntas \\
\hline $\begin{array}{c}\text { Coesão } \\
\text { Social }\end{array}$ & $\begin{array}{l}\text { 1. Na sua vizinhança, as pessoas estão dispostas a ajudar } \\
\text { seus vizinhos. } \\
\text { 2. Sua vizinhança é bem unida, isto é, as pessoas são } \\
\text { capazes de se unir em torno de interesses comuns. } \\
\text { 3. As pessoas na sua vizinhança são de confiança } \\
\text { 4. Em geral, as pessoas na sua vizinhança NÃO se dão bem } \\
\text { umas com as outras. } \\
\text { 5. As pessoas na sua vizinhança NÃO compartilham os } \\
\text { mesmos padrões culturais, de comportamento, princípios } \\
\text { éticos ou morais, entre outros. }\end{array}$ \\
\hline $\begin{array}{l}\text { Ambiente } \\
\text { para } \\
\text { atividade } \\
\text { física }\end{array}$ & $\begin{array}{l}\text { 1. Sua vizinhança oferece muitas condições para que as } \\
\text { pessoas sejam fisicamente ativas (por exemplo, possam fazer } \\
\text { caminhada, andar de bicicleta). } \\
\text { 2. Há muitas oportunidades para praticar atividades físicas ou } \\
\text { esportes em clubes, academias, ou outros espaços na sua } \\
\text { vizinhança. } \\
\text { 3. É agradável fazer caminhadas na sua vizinhança. } \\
\text { 4. As árvores da sua vizinhança dão bastante sombra. } \\
\text { 5. É fácil ir a pé aos lugares na sua vizinhança } \\
\text { 6. Frequentemente o(a) senhor(a) vê outras pessoas fazendo } \\
\text { caminhadas na sua vizinhança. } \\
\text { 7. Frequentemente o (a) senhor(a) vê outras pessoas } \\
\text { praticando atividade física na sua vizinhança (por exemplo: } \\
\text { correndo, andando de bicicleta, praticando esportes). } \\
\text { 8. O trânsito de veículos é intenso (pesado) na sua } \\
\text { vizinhança. } \\
\text { 9. É necessário atravessar muitas ruas movimentadas para } \\
\text { fazer caminhadas na sua vizinhança. }\end{array}$ \\
\hline $\begin{array}{c}\text { Disponibilida- } \\
\text { de de } \\
\text { alimento } \\
\text { saudável }\end{array}$ & $\begin{array}{l}\text { 1. Encontra-se grande variedade de frutas, verduras e } \\
\text { legumes frescos à venda próximo à sua residência. } \\
\text { 2. As frutas, verduras e legumes frescos à venda próximo à } \\
\text { sua residência são de boa qualidade. } \\
\text { 3. Encontra-se uma grande variedade de alimentos com baixo } \\
\text { teor de gordura (isto é, light/diet) à venda próximo à sua } \\
\text { residência. } \\
\text { 4. Existem muitos lugares para lanches e refeições rápidas } \\
\text { ("fast food") próximo à sua residência. }\end{array}$ \\
\hline
\end{tabular}




\begin{tabular}{|c|c|}
\hline Segurança & $\begin{array}{l}\text { 1. O(a) senhor(a) se sente seguro(a) andando de dia ou de } \\
\text { noite na sua vizinhança. } \\
\text { 2. A violência é um problema na sua vizinhança. } \\
\text { 3. Sua vizinhança é segura em relação a crimes. }\end{array}$ \\
\hline $\begin{array}{l}\text { Violência } \\
\text { percebida }\end{array}$ & $\begin{array}{l}\text { 1. Nos ÚLTIMOS } 6 \text { MESES, com que frequência houve } \\
\text { brigas que tenham envolvido o uso de armas na sua } \\
\text { vizinhança? } \\
\text { 2. Nos ÚLTIMOS } 6 \text { MESES, com que frequência houve } \\
\text { discussão violenta entre vizinhos? } \\
\text { 3. Nos ÚLTIMOS } 6 \text { MESES, com que frequência houve } \\
\text { briga entre gangues (grupos ou facções rivais)? } \\
\text { 4. Nos ÚLTIMOS } 6 \text { MESES, com que frequência houve } \\
\text { violência sexual ou estupro? } \\
\text { 5. Nos ÚLTIMOS } 6 \text { MESES, com que frequência houve } \\
\text { roubo ou assalto? }\end{array}$ \\
\hline $\begin{array}{c}\text { Vitimização } \\
\text { pessoal }\end{array}$ & $\begin{array}{l}\text { Alguém já praticou violência (assalto, briga, violência sexual } \\
\text { ou sequestro) contra o(a) senhor(a) ou contra algum morador } \\
\text { de sua residência, nessa vizinhança, durante o tempo em que } \\
\text { o(a) senhor(a) mora nesse local? }\end{array}$ \\
\hline
\end{tabular}

